\title{
Bi-steric mTORC1-selective Inhibitors activate 4EBP1 reversing MYC-induced tumorigenesis and synergize with immunotherapy
}

\section{Authors:}

11 


\section{Abstract}

The MYC oncogene is causally involved in the pathogenesis of most types of human cancer but it remains therapeutically untargeted. The mTORC1 protein complex regulates cap-dependent translation through 4EBP1 and S6K and thereby, downstream MYC protein expression. However, to date, agents such as rapalogs that selectively target mTORC1 (as compared to mTORC2) fail to reactivate 4EBP1 and thus, to block MYC in vivo. In contrast, agents that nonselectively inhibit both protein complexes of the mTOR pathway, mTORC1 and mTORC2, can activate 4EBP1, but often suffer from a lack of tolerability including in vivo hepatotoxicity and immunosuppression. Here, we report the anti-tumor activity of bi-steric mTORC1-selective inhibitors, including Revolution Medicines' clinical candidate RMC-5552, that potently and selectively target mTORC1 over mTORC2. In an autochthonous transgenic mouse model of MYCamplified and MYC-driven hepatocellular carcinoma (HCC), representative bi-steric mTORC1-selective inhibitors suppress translation initiation via activation of 4EBP1, thereby suppressing MYC protein expression and blocking tumor growth. Furthermore, in human HCC samples, the low levels of 4EBP1 and MYC is correlated with immune reactivation. Immunohistochemistry, CIBERSORT, and CODEX reveal that selective mTORC1 inhibition results in activation of both CD4+ T cell- and NKp46+ NK cellmediated immune surveillance. Moreover, bi-steric mTORC1-selective inhibitors synergize with a-PD-1 to induce sustained tumor regression, with immune cell degranulation and release of perforins and granzyme B. These agents also exhibit anti-tumor activity in human patient-derived xenografts of HCC, colorectal cancer, head and neck cancer, and ovarian cancer harboring genomic amplifications in MYC. We infer that selective mTORC1 inhibition is a potential therapeutic strategy to drive effective MYC inactivation in cancer, and the consequent restoration of immune surveillance against neoplasia. 


\section{Introduction}

The mammalian target of rapamycin (mTOR) pathway is frequently involved in the pathogenesis of human tumorigenesis including cancers of the liver, lung, kidneys, colon, prostate, and breast ${ }^{1,2,3}$. The mTOR pathway contributes to tumorigenesis by inducing cellular proliferation and survival ${ }^{4}$. mTOR forms two protein complexes: $\mathrm{mTORC} 1$ and $\mathrm{mTORC}^{1-3}$. $\mathrm{mTORC} 1$ responds to growth and stress factors resulting in the phosphorylation of two proteins, 4E-binding protein 1 (4EBP1) which becomes inactivated and serine/threonine 6 kinase (S6K) which becomes activated. mTORC2 responds to growth factors resulting in the phosphorylation of Ak strain transforming (AKT) protein which becomes activated (Fig. 1a, left). mTORC1 induces downstream protein translation while mTORC2 induces downstream transcription and prevents protein degradation of different oncogenes including MYC (Fig. 1a, left).

Therapeutics against the mTOR pathway have been suggested as a strategy to target MYC-driven cancers $^{5-7}$. MYC is one of the most commonly activated human oncogenes but it is yet to be directly targeted with a therapeutic agent ${ }^{8}$. MYC contributes to the pathogenesis of over $70 \%$ of human cancers and it is amplified in about $15 \%$ of hepatocellular carcinomas (HCC) $)^{8,9}$. Experimentally, inhibiting MYC results in tumor regression associated with immune reactivation ${ }^{8}$. The mTOR pathway is also commonly activated in cancer significantly increasing MYC protein expression ${ }^{1,10-13}$. Genetic or pharmacological inhibition of the mTOR pathway can reduce MYC-driven tumorigenesis in multiple mouse models ${ }^{1,10-13}$. The two mTOR complexes, mTORC1 and mTORC2, together regulate MYC as well as RAS, MAPK, and $\mathrm{PI} 3 \mathrm{~K} / \mathrm{AKT}^{1,12,14}$. mTORC1 regulates MYC protein translation through the phosphorylation and inactivation of $4 \mathrm{EBP} 1^{10,15}$ (Fig. 1a, left). However, the first generation mTORC1-selective allosteric inhibitor Rapamycin has a minimal effect on inhibiting 4EBP1 phosphorylation which then results in a modest decrease of MYC levels in vivo ${ }^{1,16}$. At tolerated doses, the nonselective dual mTOR kinase inhibitor MLN0128 transiently reduces p4EBP1, and as a result MYC levels, causing reduced tumor growth in preclinical models ${ }^{1,16}$. Altogether, existing mTOR pathway inhibitors including Rapamycin and MLN0128 fail to sufficiently activate 4EBP1 in vivo, and therefore cannot sufficiently decrease MYC protein levels ${ }^{17}$ to drive tumor regression (Fig. 1a, middle). 
To date, mTOR inhibitors have limited clinical benefit in cancer patients. Everolimus, a first generation Rapamycin analog, effectively inhibits S6K phosphorylation downstream of mTORC1, but does not inhibit 4EBP1 phosphorylation, which may explain its limited therapeutic effect ${ }^{18}$ (Fig. 1a, middle). Second generation nonselective mTOR kinase inhibitors, such as MLN0128, can inhibit p4EBP1 but the feasible dose and clinical efficacy of these agents is limited ${ }^{19}$ by various toxicities, including hepatotoxicity and immunosuppression ${ }^{20,21}$. The inhibition of both mTORC1 and mTORC2 causes hepatotoxicity by increasing reactive oxygen species $^{22}$ and immunosuppression characterized by hyposplenism and reduced activation and proliferation of T and natural killer (NK) cells ${ }^{18,23-31}$.

Given the importance of the mTORC1 pathway in the progression of MYC-driven cancers, we hypothesized that a potent and selective mTORC1 inhibitor that enables the activation of 4EBP1 would lead to more robust inhibition of MYC protein expression, but without hepatotoxicity or immunosuppression (Fig. 1a, right). Revolution Medicines has developed third-generation bi-steric selective inhibitors of $\mathrm{mTORC} 1$ with a rapamycin-like core moiety covalently linked to an mTOR activesite inhibitor ${ }^{32,33}$ (Fig. 1b, left). Here, we show that these agents suppress both S6K and 4EBP1 phosphorylation, and deplete MYC protein expression in vivo in an autochthonous conditional transgenic mouse model of MYC-driven $\mathrm{HCC}^{34,35,36}$. Furthermore, the bi-steric mTORC1-selective inhibitors demonstrate significant anti-tumor activity as monotherapy in our MYC-driven HCC mouse model without causing hepatotoxicity or immunosuppression. Importantly, RMC-5552, a bi-steric selective mTORC1 inhibitor currently in Phase 1 (NCT04774952), exhibits anti-tumor activity in human patient-derived xenografts (PDXs) preclinical models of HCC, colorectal, head and neck, and ovarian cancer with MYC amplifications. We also show that bi-steric mTORC1-selective inhibitors restore tumor immune surveillance and synergize with $\alpha-P D-1$ immune checkpoint therapy in the MYC-driven HCC mouse model. Hence, we provide proof-of-principle that selective mTORC1 pharmacological inhibition can effectively target MYC-driven cancers, combine with immune checkpoint inhibition, and induce sustained tumor regression in the preclinical setting. 


\section{Materials and Methods:}

108 Transgenic mice: Animals were identically raised and housed at Stanford University following 109 Administrative Panel on Laboratory Animal Care (APLAC) guidelines and procedures. The Tet-off system 110 transgenic lines used for conditional MYC expression and oncogene addiction have been previously described ${ }^{34-36}$. The Lap-tTA/Tet-O-MYC transgenic mice develop hepatocellular carcinoma within four to five weeks upon removal of doxycycline from 4 week old mice, whereby there is induced expression of the MYC transgene ${ }^{36}$. Mice genotypes were confirmed by PCR of genomic DNA from animal tails.

Cell culture: The Lap-tTA/Tet-O-MYC HCC tumor-derived cell line EC4 was grown in DMEM cells were treated for 48 hours with $20 \mathrm{ng} / \mathrm{mL}$ of doxycycline ${ }^{37}$.

Cell viability assay: A total of 3,000 MYC-driven HCC EC4 cells were plated in clear-bottom black 96-well plates, allowed to attach overnight and treated with different concentrations of a vehicle control, RapaLink-1, RMC-462732, or RMC-6272, or doxycycline for 48 hours. Upon treatment completion, EC4 cells were incubated with $5 \mathrm{mg} / \mathrm{ml}$ MTT reagent for 3.5 hours followed by addition of MTT solvent ${ }^{38}$ according to manufacturer's instructions (Abcam). 96-well plates were rocked for 15 minutes and read at an absorbance at $590 \mathrm{~nm}$ using a SpectraMax Paradigm Multi-Mode Detection Platform plate reader (Molecular Devices).

128 Cell growth assay: We plated 3,000 EC4 cells in a 96-well plates and treated with different concentrations of a vehicle control, RapaLink-1, RMC-4627, or RMC-6272, or doxycycline for 48 hours. Upon treatment completion, a CellTiTer Glow assay was performed according to manufacturer's instructions (Promega). 
132 a white opaque 96-well plate and luminescence was read using a SpectraMax Paradigm Multi-Mode 133 Detection Platform plate reader (Molecular Devices).

Real-time quantitative PCR: We seeded 6-well plates with EC4 cells at $80 \%$ confluency and treated with 136 a vehicle control, doxycycline at $20 \mathrm{ng} / \mathrm{mL}$ (MYC Off), or the inhibitors RapaLink-1, RMC-4627, or RMC1376272 at $100 \mathrm{nM}$ for 48 hours at $5 \% \mathrm{CO}_{2}$ in a $37^{\circ} \mathrm{C}$ incubator. EC4 cells were collected and pelleted, and 138 RNA was isolated using the RNeasy Plus Mini Kit (Qiagen) according to manufacturer's instructions. Equal amounts of RNA were used to synthesize cDNA using SuperScriptlll (ThermoFisher) and real-time quantitative PCR was performed using SYBR Green pPCR kit (Roche) using a HT7900 Real-Time PCR system with QuantStudio12K Flex software (Applied Biosystems). Each sample was run in triplicates and raw data was processed using the cycle threshold method were samples were normalized using UBC.

The primers used in RTqPCR analyses were: human c-MYC-forward primer 5'CTGCGACGAGGAGGAGAACT-3' and reverse primer 5'-GGCAGCAGCTCGAATTTCTT-3'; mouse cMYC-forward primer 5'-TCTCCATCCTATGTTGCGGTC-3' and reverse primer 5'TCCAAGTAACTCGGTCATCATCT-3'; mouse UBC—-forward primer 5'-ACCCAAGAACAAGCACAAGG-

3' and reverse primer 5'-AGCCCAGTGTTACCACCAAG-3'; mouse APEX1-forward primer 5'ACGGGGAAGAACCCAAGTC-3' and reverse primer 5'-GGTGAGGTTTTCTGATCTGGAG-3'; mouse Srm-forward primer 5'-ACATCCTCGTCTTCCGCAGTA-3' and reverse primer 5'GGCAGGTTGGCGATCATCT-3'; mouse Cct3-forward primer 5'-GGACCTGCTTGGGACCTAAAT-3' 152 and reverse primer 5'-CGGGATGCTGGACTTGAATC-3'; and, mouse GNL3—forward primer 5'CAGGATGCTGACGATCAAGAAA-3' and reverse primer 5'-CAGATGGCTTACCTGCTGTTG-3'.

Western blots: One million EC4 cells were plated on 6-well plates and treated with DMSO as a vehicle control, doxycycline at $20 \mathrm{ng} / \mathrm{mL}$ (MYC Off), or the inhibitors RapaLink-1, RMC-4627, or RMC-6272 at 0, 10 , or $100 \mathrm{nM}$ for 48 hours. Following treatment, cells were pelleted and stored at $-80^{\circ} \mathrm{C}$ until 
(D84C12) \#5605 and alpha tubulin (DM1A) Mouse mAb \#3873 from Cell Signaling Technologies-were previously described by our group ${ }^{39,40}$. Antibodies to detect p-4EBP1 (T37/46) (236B4) \#2855, 4EBP1 (53H11) \#9644, p-p70 S6K (T389) \#9205, p70 S6 Kinase (49D7) \#2708, and ß-actin (8H10D10) \#3700 were all from Cell Signaling Technologies. Western blot bands were quantified using ImageJ as 163 previous described ${ }^{41}$.

Size Separation "SimpleWestern" Nanoimmunoassay (NIA) ${ }^{42}$ : Simple Western 12-230 kDa size-assays were carried out using Peggy Sue ${ }^{\mathrm{TM}}$ analyzer (Protein Simple, San Jose, CA, USA). First, we prepared antibodies, the detection solution (equivalent volumes of luminol and peroxide), stacking and separation matrices, according to the manufacturer's preparation template. After centrifugation at 1000 xg during 5 171 minutes, the 384-microwell plate was loaded into Peggy Sue ${ }^{\mathrm{TM}}$ platform. Then, the complete automated workflow, ranging from samples' uptake on the plate to the chemiluminescent detection and quantification of proteins, was launched. First, samples were loaded into 12 individual single-use capillaries per cycle (1 cycle comprising 11 samples and 1 ladder), followed by a size-based separation (45 minutes in our study). A detection of fluorescence of standards, mixed with every sample, ensured a control of 177 Incubations of primary (From Cell Signaling Technologies antibodies for c-MYC (D84C12) Rabbit mAb \#5605, pS6RP (Ser235/236) (D57.2.2E) XP® Rabbit mAb \#4858, p4EBP1 (Thr37/46) (236B4) Rabbit mAb \#2855 and pAKT (Ser473) antibody \#9271). Antibodies for beta macroglobulin [EP2978Y] (ab75853) are from Abcam) and secondary antibodies were both 30 minutes in our protocol. Finally, a chemiluminescent detection of target proteins was automatically performed with different exposure times, ranging from 4 to 512 seconds. Target proteins' sizes were determined for each sample in comparison

183 to the migration of the size markers of the ladder. Heights and areas of all detected peaks were 184 systematically calculated on Compass for Simple Western® software, version 4.1.0, using the high dynamic range exposure time. 
Charge Separation Nanoimmunoassay (NIA): NIA is a highly sensitive capillary-based isoelectric focusing method that uses antibody detection to quantify protein isoforms as well as characterize posttranslational protein modifications, such as phosphorylation. Charge separation analyses were performed according to the Protein Simple user manual. Tissue homogenate samples were lysed with Bicine/CHAPS Lysis Buffer (Protein Simple, San Jose, CA, USA) containing phosphatase and protease inhibitors. Lysates were mixed with ampholyte premix and fluorescently labeled isoelectric point (pl) standards for analysis on the Peggy Sue® system (Protein Simple) according to the manufacturer's instructions. In brief, a mixture of $0.1 \mu \mathrm{g} / \mu \mathrm{L}$ cell lysate, $1 \times$ fluorescent pl standard ladder 1 , and $1 \times$ premix electrophoresis was carried out at 21,000 microwatts for 40 minutes. The separated proteins were immobilized to the capillary wall by exposing to UV light for 100 seconds at instrument default setting. Immunoprobing was done using primary antibodies (ERK1/2 (Millipore), diluted 1:300 and 4EBP1 (Cell Signaling technologies) diluted 1:50, or Beta-2-microglobulin (Abcam) diluted 1:100) and then probed with horseradish peroxidase (HRP)-conjugated, goat anti-rabbit or goat anti-mouse secondary antibodies (Protein Simple). All antibody incubation and wash steps were programmed and performed automatically in the Peggy Sue® system; the incubation time was 2 hours for primary antibodies and 1 hour for secondary antibodies. A 1:1 Luminol and peroxide (Protein Simple) mixture was then added to generate chemiluminescent light, which was captured by a charge-coupled device camera. The digital image was analyzed and quantified with Compass for Simple Western® software, version 4.1.0 (Protein Simple) with

In vivo RMC-6272 short-term treatment: Lap-tTA/Tet-O-MYC mice were taken off doxycycline to activate the human transgenic MYC oncogene 4 weeks after being born, then after waiting 4-5 weeks, mice were imaged using magnetic resonance imaging (MRI) every week to precisely define tumor volumes before treatment enrollment. Mice bearing HCC tumors with these volumes were assigned to one of the following peak areas corresponding with signal strength. 
213 4627, RMC-6272 or Sorafenib. For vehicle control, RMC-4627, and RMC-6272 groups, mice were given 214 one weekly intraperitoneal injection at $10 \mathrm{mg} / \mathrm{kg}$ while mice in the Sorafenib group were treated with 30 $215 \mathrm{mg} / \mathrm{kg}$ with a daily dose given via oral gavage. Doxycycline was supplemented in water at $100 \mu \mathrm{g} / \mathrm{mL}$. 216 Seven days after treatment, mice were given a second dose of their respective treatments and they were 217 euthanized at 4, 12, 24, 48 or 72 hours to grossly quantify tumor nodules, weight tumors, and collect 218 HCC tumors, plasma, and other tissues.

In vivo RMC-6272 long-term treatment: Lap-tTA/Tet-O-MYC mice were taken off doxycycline to activate the human transgenic MYC oncogene 4 weeks after being born, then after waiting 4-5 weeks, mice were imaged using MRI every week to verify tumor volume before treatment enrollment. Mice bearing HCC tumors were assigned to one of the following treatment groups: vehicle control, RMC-6272, $\alpha-P D-1$ (BioXCell Clone RMP1-14 and catalog number BE0146), or RMC-6272 with a-PD-1. Mice in the vehicle and RMC-6272 groups were treated with a weekly dose at $10 \mathrm{mg} / \mathrm{kg}$ injected intraperitoneally. Mice in + a-PD-1 group were treated with RMC-6272 at day 1 and with $\alpha-P D-1$ at the doses specified above on day 28 , mice were imaged and then given a second dose of their respective treatments and they were euthanized at 24 hours to grossly quantify tumor nodules, weight and collect HCC tumors, plasma, and other tissues.

Magnetic Resonance Imaging (MRI): MRI screening was utilized to enroll mice with similar tumor burden into randomized treatment groups and to track tumor progression throughout the treatments. MRI screening was performed using 7T small animal MRI (Agilent conversion) with a $40 \mathrm{~mm}$ Varian Millipede 236 RF coil (ExtendMR LLC, Milpitas, CA) at the Stanford Small Animal Imaging Facility as previously 237 described ${ }^{43,44}$. Briefly, animals were anesthetized with 1-3\% isoflurane and placed into the MRI scanner containing a $40 \mathrm{~mm}$ Varian Millipede RF coil (ExtendMR LLC, Milpitas, CA). ParaVision (PV6.01) was used to acquire the DICOM images. Once the DICOM images were acquired, tumor volumes were 
quantified and 3D models of tumors were constructed using Osirix image processing software (Osirix,

UCLA, Los Angeles, CA). The relative area difference of the 3D models before and after treatments were quantified using FIJI ImageJ software (NIH). Percent tumor regression was calculated by dividing the number of mice whose tumors were smaller by Day 7 compared to Day 0 to the total number of mice treated in each group and multiplying this number by $100 \%$. The same calculation was done for mice enrolled in long-term treatments. For example, regression at Day 14 was calculated by dividing the number of mice whose tumors were smaller by Day 14 compared to Day 7 to the total number of mice treated in each group and multiplying this number by $100 \%$.

Liver function and toxicity studies: $250-300 \mu \mathrm{l}$ of blood were collected via the retro-orbital route from Laphigh-density lipoprotein (HDL), low-density lipoprotein (LDL), chloride, $\mathrm{CO}_{2}$, glucose, albumin, creatinine, total protein, blood urea nitrogen (BUN), calcium, and globulin. For liver function and toxicity studies in mice treated with vehicle control, RMC-6272, $\alpha-P D-1$, or RMC-6272 + $\alpha-P D-1$, blood was collected retro-

Survival meta-analysis: To determine differences in progression free survival in HCC patients with high 262 versus low MYC signature genes and EIF4EBP1 we downloaded The Cancer Genome Atlas (TCGA) 263 Liver hepatocellular carcinoma (LIHC) RNA-seq dataset from xenabrowser.net. We then used the 264 Qlucore Omics Explorer software to run the HALLMARK_MYC_TARGETS_V2 gene set 265 (https://www.gsea-msigdb.org/gsea/msigdb/cards/HALLMARK_MYC_TARGETS_V2) and using signscore, we determined patients' samples with high and low MYC signature. From this analysis, we 
267 extracted the top and bottom 40 samples in each group, respectively. Following separation by MYC 268 signature, we used the top and bottom 20 samples in each group that had the highest and the lowest 269 levels of EIF4EBP1, respectively. We then plotted their respective overall survival, disease-free survival, 270 and progression-free survival. Similarly, to choose MYC+/EIF4EBP1+ and MYC-/EIF4EBP1- samples, 271 we selected those with the highest and lowest EIF4EBP1 within MYC high and MYC low groups getting 27214 samples per group. Within these samples per group, those samples lacking survival data were 273 excluded from the study.

Metanalysis of protein levels from HCC patients: To generate the volcano plot of protein levels using the TCGA LIHC and The Cancer Proteome Atlas (TCPA) LIHC datasets, we first segregated patients' samples into high and low MYC signature using the HALLMARK_MYC_TARGETS_V2 gene set and signscore as described above. The top and bottom 20 samples were aligned to the TPGA LIHC dataset to choose the patients for which there is protein data in the TCPA LIHC dataset. There were seven patient samples in the MYC high group and 11 patient samples in the MYC low group. We used these 18 samples to calculate the $-\log _{10} \mathrm{p}$-value and to calculate protein levels as $\log _{2}$ MYC high over MYC low which are depicted as a volcano plot.

CIBERSORT: We downloaded the TCGA LIHC RNA-seq dataset from xenabrowser.net. We then used the Qlucore Omics Explorer software to open this dataset and to run the

287 msigdb.org/gsea/msigdb/cards/HALLMARK_MYC_TARGETS_V2) to segregate patients' samples into 288 high and low MYC signature using signscore as described above. From this analysis, we extracted the 289 top and bottom 20 samples in each group, respectively. Following separation by MYC signature, we 290 plugged the RNAseq data into cibersortx.stanford.edu using the LM22 gene matrix to estimate the 291 presence of tumor infiltrating immune cell populations from HCC patient samples expressing high and low MYC signature genes. To estimate abundance of immune populations from RNA-seq data of murine 
293 HCC tumors treated with vehicle, doxycycline, RMC-4627, RMC-6272, or sorafenib, we utilized 294 CIBERSORT ${ }^{45,46}$ and TIMER2.047 using the http://timer.cistrome.org/ website.

296 CO-Detection by indexing (CODEX) antibody staining of Fresh Frozen mouse tissue: The tissue section 297 was removed from $-80^{\circ} \mathrm{C}$ and placed onto a bed of drierite absorbent beads. After 2 minutes the tissue 298 was placed in acetone for 10 minutes. The tissue was then allowed to dry for 2 minutes and hydrated in 299 hydration buffer (Akoya Biosciences) twice for 2 minutes. After hydration, tissue was fixed for 10 minutes using $1.6 \%$ paraformaldehyde (PFA) in hydration buffer. This was followed by two washes in hydration buffer and equilibration in staining buffer (Akoya Biosciences) for 20 minutes. Blocking buffer was prepared by adding N, S, J and G blocking solutions to CODEX staining buffer (Akoya Bioscience). Antibodies were then added to this blocking buffer in a 1:50 titration to make a total volume of $100 \mu \mathrm{l}$.

The antibody cocktail was added to the coverslip and staining was performed in a sealed humidity chamber at $4^{\circ} \mathrm{C}$ overnight. After staining, coverslips were washed twice in hydration buffer for a total of 4 minutes followed by fixation in storage buffer (Akoya Bioscience) with 1.6\% paraformaldehyde for 10 minutes. Coverslips were then washed thrice in PBS, followed by a 5 minute incubation in ice cold methanol on ice followed by another three 1x PBS washes. CODEX fixative solution (Akoya Bioscience) was prepared right before the final fixation step. $20 \mu \mathrm{l}$ of CODEX fixative reagent was added to $1000 \mu \mathrm{l}$ 1x PBS. $200 \mu \mathrm{l}$ of the fixative solution was added to the coverslip for 20 minutes followed by 3 washes in 1x PBS. Coverslips were then stored in CODEX storage buffer at $4^{\circ} \mathrm{C}$ until imaging (for a maximum of 2 weeks). Antibodies used in the experiment are Akoya Bioscience's validated panel for mouse fresh frozen 313 tissue.

CODEX multicycle setup and Imaging: Coverslips were taken out of $4^{\circ} \mathrm{C}$ and allowed to come to RT for $316 \quad 15-20$ minutes. The coverslips were mounted onto Akoya's custom made stage in between coverslip 317 gaskets with the tissue side facing up. The coverslips were cleaned from the bottom using a Kim wipe to 318 get rid of any salts. The tissue was stained with Hoechst nuclear stain at a 1:2000 dilution in 1x CODEX 319 buffer. A 96-well plate was used to set up the multicycle experiment with different fluorescent 
oligonucleotides in each well. Reporter stock solution was prepared containing 1:300 Hoechst stain and 1:12 dilution of assay reagent in 1x CODEX buffer (Akoya Bioscience). Fluorescent oligonucleotides (Akoya Bioscience) were added to this reporter solution at a final concentration of 1:50 in a total of 250 $\mu \mathrm{l}$ per well. A blank cycle was performed at the start and end of the experiment. Automated image 324 acquisition and fluidics was performed using Akoya's software driver CODEX Instrument Manager (CIM) 325 and the CODEX platform (Akoya Bioscience). Imaging was performed using a Keyence BZ-X800 microscope, fitted with a Nikon CFI Plan Apo 20X/0.75 objective. The navigation tool in the BZ-X software (Keyence) was used to set up the center of each of the TMA and define each region. $15 \mathrm{z}$ steps were acquired with the pitch set at 1.5 in the BZ-X software. All images were processed using the CODEX processor (Akoya Bioscience). Numbers of Ki67, CD4, CD8a, CD11c, and CD19 positive cells were manually counted using imageJ.

Patient-derived xenograft (PDX) studies: Four HCC PDXs as well as one PDX each from a colorectal cancer patient, head and neck cancer patient, and an ovarian cancer patient with MYC amplifications were grown in immunocompromised mice. Once PDXs reached a size of $200-300 \mathrm{~mm}^{3}$, they were treated with vehicle control or RMC-5552 at 3 or $10 \mathrm{mg} / \mathrm{kg} /$ week via intraperitoneal injection for 28 days. All PDX mouse studies were conducted in compliance with all applicable regulations and guidelines of the Institutitonal Animal Care and Use Committee (IACUC). Tumor volumes and body weights were measured and weighed, respectively, twice a week during the study.

For PDX studies conducted at GenenDesign, Shanghai, China, and at WuXi AppTec (Suzhou), China,

341 female BALB/c nude mice were used. For PDX studies conducted at Charles River Discovery Research 342 Services Germany GmbH, female NMRI nu/nu mice (NMRI-Foxn1nu) were used.

RNA sequencing:

345 Data Analysis: Downstream analysis was performed using a combination of programs including STAR, 346 HTseq, Cufflink and our wrapped scripts. Alignments were parsed using the Tophat program and 
347 differential expressions were determined through DESeq2/edgeR. GO and KEGG enrichment were

348 implemented by the ClusterProfiler. Gene fusion and difference of alternative splicing event were 349 detected by Star-fusion and rMATS software.

351 Reads mapping to the reference genome: Reference genome and gene model annotation files were 352 downloaded from genome website browser (NCBI/UCSC/Ensembl) directly. Indexes of the reference 353 genome was built using STAR and paired-end clean reads were aligned to the reference genome using 354 STAR (v2.5). STAR used the method of Maximal Mappable Prefix(MMP) which can generate a precise mapping result for junction reads.

Quantification of gene expression level: HTSeq v0.6.1 was used to count the read numbers mapped of each gene. And then FPKM of each gene was calculated based on the length of the gene and reads count mapped to this gene. FPKM, Reads Per Kilobase of exon model per Million mapped reads, considers the effect of sequencing depth and gene length for the reads count at the same time, and is currently the most commonly used method for estimating gene expression levels ${ }^{48}$.

Differential expression analysis: (For DESeq2 with biological replicates) Differential expression analysis between two conditions/groups (two biological replicates per condition) was performed using the DESeq2 R package (2_1.6.3). DESeq2 provide statistical routines for determining differential expression in digital gene expression data using a model based on the negative binomial distribution. The resulting $\mathrm{P}$-values were adjusted using the Benjamini and Hochberg's approach for controlling the False Discovery Rate(FDR). Genes with an adjusted P-value $<0.05$ found by DESeq2 were assigned as differentially expressed. (For edgeR without biological replicates) Prior to differential gene expression analysis, for each sequenced library, the read counts were adjusted by edgeR program package through one scaling 371 normalized factor. Differential expression analysis of two conditions was performed using the edgeR $\mathrm{R}$ 372 package (3.16.5). The $\mathrm{P}$ values were adjusted using the Benjamini \& Hochberg method. Corrected P373 value of 0.05 and absolute foldchange of 1 were set as the threshold for significantly differential 
expression. The Venn diagrams were prepared using the function vennDiagram in $\mathrm{R}$ based on the gene list for different group.

Correlations: To allow for log adjustment, genes with 0 FPKM are assigned a value of 0.001 . Correlation were determined using the cor.test function in R with options set alternative ="greater" and method $=$ "Spearman".

Clustering: To identify the correlation between difference, we clustered different samples using expression level FPKM to see the correlation using hierarchical clustering distance method with the function of heatmap, SOM (Self-organization mapping) and kmeans using silhouette coefficient to adapt the optimal classification with default parameter in $\mathrm{R}$.

GO and KEGG enrichment analysis of differentially expressed genes: Gene Ontology (GO) enrichment analysis of differentially expressed genes was implemented by the clusterProfiler R package, in which gene length bias was corrected. GO terms with corrected P-value less than 0.05 were considered significantly enriched by differential expressed genes. KEGG is a database resource for understanding high-level functions and utilities of the biological system, such as the cell, the organism and the ecosystem, from molecular level information, especially large-scale molecular datasets generated by genome sequencing and other high-through put experimental technologies (http://www.genome.jp/kegg/). We used clusterProfiler R package to test the statistical enrichment of differential expression genes in KEGG pathways.

PPI analysis of differentially expressed genes: PPI analysis of differentially expressed genes was based on the STRING database, which contained known and predicted Protein-Protein Interactions. For the species existing in the database (like human and mouse), we constructed the networks by extracting the target gene lists from the database. 
401 Differentially expressed gene annotation: TFCat and Cosmic database were used to annotate 402 differentially expressed genes. TFCat is a curated catalog of mouse and human transcription factors (TF) 403 based on a reliable core collection of annotations obtained by expert review of the scientific literature. 404 COSMIC is a database designed to store and display somatic mutation information and related details 405 which contains information relating to human cancers.

Data access: The high-throughput sequencing data from this study have been submitted to the NCBI Sequence Read Archive (SRA) under accession number \#\#\#.

Immunohistochemistry (IHC): HCC tumors from Lap-tTA/Tet-O-MYC mice treated with a vehicle control, 411 doxycycline, RMC-4627, RMC-6272, or sorafenib for 7 days or with a vehicle control, RMC-6272, $\alpha-P D-$ 412 1, or RMC-6272 + a-PD-1 for 28 days were immersed and fixed in 10\% formalin for $24 \mathrm{~h}$ and transferred 413 to $70 \%$ ethanol. Tissues were embedded in paraffin using standard procedures on a Tissue-TEK VIP 414 processor (Miles Scientific). From these paraffin blocks, $5 \mu \mathrm{m}$ sections were mounted on Apex superior 415 adhesive slides (Leica Microsystems) and stained as previously described ${ }^{43}$. Stained IHC sections were mounted with antifade mounting medium (Pro-Long Gold; Invitrogen) and coverslips were used to seal 417 the slides, Images from slides were acquired at $25^{\circ} \mathrm{C}$ on a Zeiss Axiovert $200 \mathrm{M}$ inverted confocal 418 microscope with a 40 Plan Neofluor objective using IP Lab 4.0 software (Scanalytics). Antibodies used 419 for IHCs include: human MYC (Millipore Sigma c-Myc (EP121)), CD4 (abcam, ab183685, EPR19514), 420 NKp46 (abcam, ab214468), NKG7 (Cell Signaling Technologies, 65507), perforin (Cell Signaling 421 Technologies, E3W4I), granzyme B (Cell Signaling Technologies, D6E9W) and the same antibodies used 422 in western blot and NIA for pS6RP, and p4EBP1.

424 Statistical analyses: Graphpad prism (GraphPad Prism software) was used to generate graphs and to 425 perform statistical analyses. These analyses were performed using an unpaired t-test assuming 426 Gaussian distribution with Welch's correction without assuming equal standard deviations for all 427 comparisons between two different treatment groups while two-way ANOVA was used to determine 
428 significance between multiple treatment groups at different time points. For PDX studies, tumor volumes 429 at the end of study were compared between control and treatment groups by two-way ANOVA Dunnet's 430 multiple comparison test. Log rank (Mantel-Cox) test was used for Kaplan-Meier survival analyses. Error 431 bars represent standard deviations (SD) or standard error of the mean (SEM) as specified in each figure 432 legend. A probability value $(\mathrm{P})$ of 0.05 or lower was considered significant and is indicated by one asterisk $433\left(^{*}\right)$ while $\mathrm{P}<0.01$ is indicated by two asterisks $\left({ }^{* *}\right), P<0.005$ is indicated by three asterisks $\left({ }^{* *}\right)$, and $\mathrm{P}$ $434<0.001$ is indicated by four asterisks $\left({ }^{* * *}\right)$. 


\section{Results:}

440 Bi-steric mTORC1-selective inhibitors potently reduce p4EBP1 in vitro:

441 The prototype bi-steric pan-mTOR inhibitor RapaLink-1 and the early bi-steric mTORC1-selective tool 442 compound RMC-4627 were previously reported ${ }^{32,33}$. Here, we tested two additional bi-steric mTORC1443 selective inhibitors: a research tool compound-RMC-6272 and Revolution Medicines' development 444 candidate-RMC-5552 (NCT04774952). Both exhibit improved potency and selectivity as compared to 445 the pan-mTOR bi-steric inhibitor RapaLink-1 and to RMC-4627 ${ }^{32,33}$ (Fig. 1b). RMC-4627, RMC-6272, and 446 RMC-5552 all selectively inhibit mTORC1 (as compared to mTORC2) and consequently decreased 447 phosphorylation of 4E-binding protein 1 (4EBP1) and serine/threonine 6 kinase (S6K) over 448 phosphorylation of Ak strain transforming (AKT) protein in several cancer cell lines in vitro (Fig. 1b, table; 449 selectivity of 13,27 , and 40 respectively).

451 The chemical structures of the bi-steric inhibitors described herein are shown in Fig. 1b. As previously 452 described, RMC-4627 contains an ether chemical handle with an alkyne terminus at the C40 position on 453 rapamycin. An azide containing PEG linker appended to a PP242-derived active-site inhibitor was then 454 coupled to the alkyne containing chemical handle via a copper-catalyzed cycloaddition ${ }^{32}$. RMC-6272 and RMC-5552 exchanged this ether chemical handle for a C40 carbamate to allow for synthetic tractability, and incorporated the XL388-derived and MLN0128-derived active site inhibitors, respectively (Fig. 1b).

Selective mTORC1 inhibition decreases MYC protein levels and inhibits tumor cell growth in vitro:

Next, we studied the ability of these inhibitors to selectively inhibit mTORC1, decrease phosphorylation of 4EBP1 and S6K, and influence MYC protein expression in the mouse tumor-derived cell line, EC4.

461 This cell line is derived from a Tet system conditional transgenic mouse model of human MYC-driven 462 HCC (see $\left.\operatorname{Ref}^{36}\right)$. In this model human MYC transgene is constitutively and specifically expressed in the 463 liver, and upon doxycycline treatment the human MYC gene is shut off (MYC Off) and human MYC protein is no longer expressed. 
We evaluated the effects of bi-steric mTORC1-selective inhibitors on the expression of human MYC and mouse Myc mRNA and MYC protein expression. First and as expected, we found that for mRNA expression of human MYC (hMYC), RMC-4627 and RMC-6272 did not have any effect. But, exposure to each of these inhibitors was associated with a small increase in the levels of endogenous mouse Myc mRNA (mMYC) (Fig. 1c). This was suggestive that although MYC transgene mRNA expression was unchanged, these inhibitors were affecting the human MYC transgene protein expression, since MYC protein levels are inversely correlated with mRNA levels via feedback regulation of endogenous mouse Myc mRNA transcription ${ }^{35,36}$. Indeed, when we measured MYC protein expression, we found that RMC6272 and RMC-4627 reduced MYC levels relative to the control, consistent with the relative potency for p4EBP1 seen above (Fig. 1d, 92\% and 57\%, respectively). We found evidence that the decrease in MYC protein was associated with a decrease in its transcriptional activity on target genes, since RMC-6272, more than RMC-4627, reduced the expression of MYC target genes Apex1, Srm, Cct3, and Gn/349-53 relative to the control (Fig. 1e, $50 \%$ and $30 \%$, respectively).

Next, we examined the ability of the bi-steric mTORC1 inhibitors to selectively inhibit mTORC1 versus mTORC2 in EC4 cells, by measuring phosphorylation of 4EBP1, S6K, and AKT. Treatment with $100 \mathrm{nM}$ of RMC-4627 32 and RMC-6272 potently reduced p4EBP1 (55\% and 46\%, respectively) and pS6K (29.5\% and $30 \%$, respectively) but weakly decreased AKT phosphorylation (33\% and 13\%, respectively; Fig. 1f). These findings are consistent with the selectivity observed in MDA-MB-468 cells (Fig. 1b), which shows that RMC-6272, had a 27-fold higher selectivity for mTORC1 over mTORC2, and RMC-4627 was selective by 13-fold in MDA-MB-468 cells (Fig. 1b). Thus, RMC-6272 and RMC-4627 decreased p4EBP1, pS6K, and MYC protein levels in the EC4 murine HCC cell line.

We also examined the ability of RMC-6272 and RMC-4627, to inhibit in vitro tumor cell growth and viability. Both RMC-6272 and RMC-4627 effectively reduced the proliferation and viability of MYC-driven EC4 cells compared to the vehicle group (Fig. 1g-1h). Of note, RMC-6272 showed higher potency than RMC-4627 at decreasing proliferation and viability of EC4 cells (Fig.1g-1h). 
494 High levels of both 4EBP1 and MYC predict poor survival outcomes in human patients with HCC:

495 Our in vitro results support the notion that mTORC1 and its regulation of MYC expression through 496 4EBP1 ${ }^{10,15}$ are key nodal points for HCC cell growth. Next, we performed meta-analyses of The Cancer 497 Genome Atlas (TCGA) and The Cancer Proteome Atlas (TCPA) Liver hepatocellular carcinoma (LIHC) 498 datasets. In human HCC, increased expression of genes in the hallmarks of MYC datatset (MYC $\left.{ }^{\mathrm{Sig}}\right)$ was associated with high protein levels of mTORC1 factors-mTOR, RAPTOR, 4EBP1, P70S6K1, and S6, in line with findings from others ${ }^{1,11-13}$ (Fig. 2a). HCC patients with tumors expressing low MYC ${ }^{\text {Sig }}$ showed better overall survival, progression-free survival, and disease-free survival than patients with high $\mathrm{MYC}^{\text {Sig }}$, and high EIF4EBP1 levels further drove poorer survival in the low MYC Sig patients (Fig. 2b and Sup. Fig. 1a-1b). The combination of low MYC $\mathrm{Mig}^{\mathrm{and}}$ low EIF4EBP levels in human HCC tumors is associated with the best survival in HCC patients from our meta-analysis based on MYC ${ }^{\text {sig }}$ and EIF4EBP1 expression

505

506

507 levels.

Selective mTORC1 inhibition depletes p4EBP1 and MYC protein levels in vivo:

We evaluated the ability of RMC-4627 and RMC-6272 to activate 4EBP1 and reduce MYC protein in vivo. Transgenic mice with MYC-driven HCC were treated with RMC-4627 or RMC-6272, each at a single dose of $10 \mathrm{mg} / \mathrm{kg} /$ week. As assessed by nanoimmmuno assay $\left(\mathrm{NIA}^{42}\right)$ and immunohistochemistry (IHC), RMC-6272 treatment depleted MYC protein in tumors similarly to the MYC Off control group in vivo while RMC-4627 reduced MYC levels by $50 \%$ as assessed using both assays (Fig. 2c-2d and Sup. Fig. 2a2b). Also, RMC-6272 depleted p4EBP1 and pS6RP (as a surrogate marker for pS6K), but not pAKT, in MYC-driven tumors while RMC-4627 reduced p4EBP1 and pS6RP proteins by $60 \%$ and $80 \%$, respectively, as assessed by NIA and IHC (Fig. 2c-2d and Sup. Fig. 2a-2b). Thus, RMC-4627 and RMC6272 retain selectivity for mTORC1 over mTORC2 in vivo. To benchmark these results, we also examined the effect of the pan-RTK inhibitor sorafenib ${ }^{54}$, currently approved for HCC treatment, on reducing protein levels of MYC and mTOR substrate phosphorylation. Sorafenib treatment did not reduce MYC, p4EBP1 or pS6RP levels in vivo but decreased pAKT levels by 50\% (Fig. 2c-2d and Sup. Fig. 2a). 
521 Furthermore, using charge separation based NIA ${ }^{42}$, RMC-4627 and RMC-6272 reduced the levels of 522 multiple heavily phosphorylated 4EBP1 isoforms without affecting levels of unphosphorylated 4EBP1 523 (Up-4EBP1) (Fig. 2e-2f). Notably, neither RMC-4627 nor RMC-6272 affected phosphorylation of ERK1/2, 524 which are not regulated by mTORC1 (Sup. Fig. 2c-2d). Thus, RMC-6272, and (to a lesser extent) RMC525 4627, selectively reduced p4EBP1 protein levels in vivo resulting in a decrease in MYC protein.

$\underline{\text { mTORC1 inhibition potently reduces MYC transcriptional activity in vivo: }}$

We next examined the effect of RMC-4627 and RMC-6272 on downstream MYC transcriptional activity in vivo. Consistent with the effects on MYC protein levels above, RMC-6272 reduced the expression of MYC target genes and led to a relative enrichment score (ES) of -0.85 , which is similar to MYC Off, while RMC-4627 also reduced their expression but to a lesser extent than RMC-6272 (Fig. 2g and Sup. Fig. 3). MYC-regulated genes can also be regulated by other oncogenes (WNT, RAS, HRAS, KRAS, BRAF, and PI3K) overexpressed in $\mathrm{HCC}^{4}$. To determine the specificity of RMC-6272 and RMC-4627 for MYC, we asked if these treatments decreased the expression of genes regulated by other oncogenes in HCC, via GSEA. RMC-6272, and similarly RMC-4627, did not significantly downregulate the expression of genes regulated by WNT, NRAS, HRAS, KRAS, BRAF, and PI3K (Sup. Fig. 4). Thus, RMC-4627 and to a greater extent RMC-6272 selectively reduced MYC transcriptional activity in vivo without influencing the transcriptional activity of other oncogenes known to be overexpressed in HCC. These results are consistent with the notion that MYC as an oncogene relying on cap-dependent translation is susceptible to inhibition of p4EBP1 ${ }^{15}$, which was achieved by RMC-4627 and RMC-6272.

Next, we evaluated whether the selectivity of RMC-4627 and RMC-6272 for mTORC1 versus mTORC2 543 resulted in selective regulation of downstream genes using ES and GSEA in vivo. RMC-6272, and to a 544 lesser extent, RMC-4627 reduced the expression of genes commonly overexpressed upon mTORC1 545 activation without affecting expression of genes upregulated by mTORC2 activation (Fig. 2h-2i and Sup. 546 Fig. 3). Notably, the tyrosine kinase inhibitor approved for treatment of HCC, sorafenib ${ }^{54}$, decreased 
547 expression of mTORC2-regulated genes without affecting the expression of genes upregulated by

548 mTORC1 activation (Fig. 2h-2i and Sup. Fig. 3), As expected, sorafenib but not RMC-6272 or RMC-4627,

549 decreased the expression of MAPK-regulated genes (Fig. 2j and Sup. Fig. 3). Thus, bi-steric mTORC1-

550 selective inhibitors, but not sorafenib, selectively reduced mTORC1 activation without affecting mTORC2

551 activation in vivo.

553 Bi-steric mTORC1 inhibitor treatment results in sustained inhibition of p4EBP1 and MYC in vivo:

554 We evaluated the in vivo durability of response for RMC-4627 and RMC-6272 to reduce p4EBP1 and 555 MYC levels following administration of a single dose. The kinetics of MYC, p4EBP1, pS6RP, and pAKT 556 protein inhibition by mTORC1 inhibitors was measured using NIA and IHC. As assessed by NIA, RMC5576272 rapidly reduced p4EBP1 and pS6RP by 4 hours followed by a significant reduction in MYC protein 558 levels by 12 hours reaching its peak of inhibition by 24 hours. At this time point, RMC-6272 decreased 559 MYC levels to $2 \%$, p4EBP1 levels to $1 \%$ and pS6 levels to $1 \%$. Levels of MYC, p4EBP1, and pS6RP 560 increased slightly after 24 hours but remained relatively low (6\%, 5\%, and $2 \%$, respectively) out to 72 561 hours (Fig. 2k-2m). RMC-4627 reduced p4EBP1, pS6RP, and MYC levels by 12 hours and reach its peak 562 of inhibition by 24 hours decreasing MYC to 25\%, p4EBP1 to $10 \%$ and pS6 to $2 \%$. By 72 hours post RMC-4627 treatment, p4EBP1 and MYC levels recovered to 70\% and 55\%, respectively (Fig. 2k-2m).

These results were corroborated using immunohistochemistry (Sup. Fig. 5). Importantly, pAKT levels were not reduced over a 72-hour period following treatment with either RMC-6272 or RMC-4627 (Fig. 2n). In contrast, sorafenib treatment did not reduce protein levels of MYC, p4EBP1, or pS6RP as assessed by NIA and IHC (Fig. 2k-2n and Sup. Fig. 5). Our findings show that bi-steric mTORC1-selective inhibitors exhibit potent, selective, and durable in vivo suppression on p4EBP1 and MYC.

Selective mTORC1 inhibitors induce tumor regression in MYC-amplified human PDX models:

571 In order to translate our findings with RMC-4627 and RMC-6272, RMC-5552 (clinical development 572 candidate currently in Phase 1, NCT04774952) was tested in four patient-derived xenograft (PDX) 573 models of human HCC with different MYC copy number variations (CNV) ranging from 2 (normal) to 6.42 
574 (Fig. 3a-3d). RMC-5552 at two different doses did not significantly reduce tumor growth of an HCC tumor 575 with normal $M Y C$ copy number $(\mathrm{CNV}=2)($ Fig. 3a). In contrast, human HCC PDX tumors with genomic 576 amplification of $M Y C$ indicated by high CNV were sensitive to RMC-5552 which reduced tumor growth at 577 tested doses (Fig. 3b-3d). Furthermore, we tested whether we could extend the proof of concept that 578 selective mTORC1 inhibition via RMC-5552 observed in MYC-driven HCC models could reduce tumor 579 growth in additional models of MYC-driven human cancers. Consistent with our findings in multiple HCC 580 models, RMC-5552 was effective at reducing tumor growth of multiple human cancer models with genomic $M Y C$ amplifications including colorectal cancer (CRC), head and neck squamous cell cancer, and ovarian cancer in a dose-dependent manner (Sup. Fig. 6). Thus, the selective mTORC1 inhibitor RMC-5552 exhibited anti-tumor activity across a variety of human PDX models with genomic MYC amplification.

Bi-steric mTORC1 selective inhibitors induce regression of autochthonous MYC-driven HCC tumors in

Vivo:

Given the significant ani-tumor activity of RMC-5552 and the profound effects of RMC-4627 and RMC-

6272 treatment on $\mathrm{mTORC} 1$ inhibition and consequent effects on MYC; we examined the anti-tumor activity of these bi-steric mTORC1 inhibitors following a single dose in our MYC-driven HCC conditional transgenic mouse model ${ }^{36}$, as compared to sorafenib dosed daily for one week (Fig. 3e). Consistent with on-target pathway suppression, as well as with MYC protein reduction described above, RMC-4627 and RMC-6272 treatment reduced the number of liver tumor nodules by $28 \%$ and $68 \%$ respectively, compared to the vehicle control, while sorafenib had no effect (Fig. 3f). RMC-6272, similar to the MYC 595 Off group, and RMC-4627 to a lesser extent, induced regression of MYC-driven HCC tumors while the 596 control vehicle and sorafenib did not, as assessed by tumor volume change over time calculated from 2D $597 \mathrm{MRI}$ images, and tumor area change over time calculated from 3D reconstructions of MRI images (Fig. $5983 g-3 i$ and Sup. Table 1). RMC-4627 and RMC-6272 both reduced total tumor burden over a period of one week as measured by percent liver weight/body weight whereas sorafenib had no effect (Fig. 3j). 600 Furthermore, a single dose of RMC-6272 induced tumor regressions in $80 \%$ of treated mice while RMC- 
6014627 induced regessions in $20 \%$ of mice compared to sorafenib which did not induce tumor regression

602 at all (Fig. 3k). As expected, MYC Off resulted in 100\% tumor regression in all animals (Fig. 3k).

603 Impressively, the bi-steric mTORC1 inhibitor RMC-6272 induced tumor regressions in transgenic mice 604 with large tumor burden (Sup. Fig. 7). Therefore, bi-steric mTORC1-selective inhibitor treatment showed 605 remarkable anti-tumor activity and drove significant tumor regression in a MYC-driven HCC model 606 following a single dose.

607

608

Selectively inhibiting mTORC1 is well tolerated and does not induce hepatotoxicity or hyposplenism in mice harboring MYC-driven HCC:

In preclinical and clinical experiments, treatment with nonselective mTOR inhibitors was associated with

611 body weight loss, hepatic toxicity ${ }^{22}$, and immunosuppression characterized by hyposplenism ${ }^{31}$. In

612 contrast, a single dose of RMC-6272 or RMC-4627 did not result in body weight reduction while exhibiting 613 anti-tumor activity in a MYC-driven HCC model whereas sorafenib reduced body weight by $8 \%$ (Sup. Fig. 614 8a). Importantly, RMC-6272 and RMC-4627 did not induce hepatoxicity as measured through normal 615 circulating levels of liver enzymes: ALT, AST, alkaline phosphatase, bilirubin, and GGT (Sup. Fig. 8b). 616 These mTORC1 inhibitors did induce a modest decrease in circulating triglycerides and low-density 617 lipoprotein (LDL) (Sup. Fig. 8c-8q). Neither RMC-6272 nor RMC-4627 were associated with 618 hyposplenism while sorafenib reduced spleen sizes by $32 \%$ (Sup. Fig. 9). Thus, single dose 619 administration of RMC-6272 and RMC-4627 was not associated with weight loss, hepatotoxicity or 620 hyposplenism while driving marked anti-tumor activity in a MYC-driven tumor model.

Downregulation of MYC signaling and EIF4EBP1 in human $\mathrm{HCC}$ is predictive of immune reactivation:

623 First-generation non-selective mTOR inhibitors are associated with immune suppression ${ }^{22,31}$. But, since 624 MYC inactivation causes immune reactivation ${ }^{8}$, we first evaluated if MYC signaling (MYC $\left.{ }^{S i g}\right)$ and 625 EIF4EBP1 levels predict immune activation in human HCC tumors using CIBERSORT ${ }^{45}$. Human HCC 626 tumors expressing low MYC ${ }^{\text {Sig }}$ and low EIF4EBP1 versus those expressing high MYC ${ }^{\text {Sig }}$ and high 627 EIF4EBP1 levels had lower numbers of inactivated M0 macrophages and immune inhibitory $\mathrm{T}_{\text {reg }}$ cells 
and increased activated DCs, CD4+ memory T cells, and activated NK cells (Sup. Fig. 10). Thus, low $\mathrm{MYC}^{\text {Sig }}$ and low EIF4EBP1 levels are associated with immune activation in human HCC through the recruitment of DCs, T cells, and NK cells. Based on these data, we subsequently evaluated whether RMC-6272 treatment can restore anti-tumor immunity in MYC-driven HCC tumors.

Selective mTORC1 inhibition induces immune activation in MYC-driven tumors:

We focused our continued studies on RMC-6272 because it durably suppressed MYC protein levels in vivo. We examined how RMC-6272 treatment influenced the immune state in vivo in our MYC-driven HCC mouse model through three methods: GSEA and GO Term enrichment analyses of RNA-seq data, CIBERSORT ${ }^{46}$ and TIMER2.047, and CO-Detection by indexing (CODEX) ${ }^{55}$. We compared RMC-6272 with sorafenib and the MYC Off condition in select analyses as warranted.

By GSEA, RMC-6272, similarly to MYC Off, upregulated genes involved in immune system expansion and function by 24 hours which was durable through 72 hours (Fig. 4a-4c). Conversely, sorafenib did not induce genes involved in immune system expansion at any time point tested (Fig. 4a-4c). By GO Term enrichment analyses, RMC-6272 induced pathways that regulate DNA replication, cell cycle progression, and ribosome biosynthesis by 24 hours (Fig. 4d) and by 48 and 72 hours, enrichment in inflammatory responses including leukocyte migration and activation (Fig. 4e and 4f). Thus, RMC-6272 treatment may induce immune cell migration, activation, and expansion.

Second, by CIBERSORT ${ }^{46}$ and TIMER2.047 we found RMC-6272, similar to MYC Off, decreased immune-suppressive subsets including $\mathrm{T}_{\text {reg }}$ cells, M2 macrophages, and mast cells in our MYC-driven HCC model (Fig. 4g). Furthermore, RMC-6272 increased memory B cells, CD4+ T cells, activated NK cells, M1 macrophages, and myeloid dendritic cells (DCs) (Fig. 4g). Moreover, treatment with sorafenib reduced memory CD4+ T cells, activated NK cells, and myeloid DCs while memory B cells increased but not to the extent of RMC-6272 or MYC Off (Fig. 4g). Notably, the abundance of $\mathrm{T}_{\text {reg }}$ cells and anti- 
654 inflammatory M2 macrophages increased upon sorafenib treatment in clear contrast to RMC-6272 655 treatment (Fig. $4 g)$.

Third, by CODEX ${ }^{55}$, RMC-6272 treatment resulted in the recruitment of CD4+ cells and CD11C+ immune cells accompanied by a decrease in Ki67+ cells (Fig. 4h-4j). In RMC-6272 and MYC Off tumors, CD4+ immune cells were found in pockets of space that are cleared of Ki67+ cells or that have cells expressing low Ki67 (Fig. 4h, white arrows). Higher numbers of tumor-recruited CD4+ immune cells can be found in tissue pockets with lower numbers of Ki67+ cells; in fact, the number of CD4+ cells are inversely correlated to the number of Ki67+ cells in MYC-driven HCC tumors (Fig. 4k). Thus, RMC-6272 induced immune activation associated with both increased CD4+ and CD11c+ immune cells at sites of nonproliferating Ki67 ${ }^{\text {low/- }}$ cancer cells.

RMC-6272 and $\alpha-P D-1$ cooperate to induce sustained tumor regression:

Since RMC-6272 enhanced immune activation, we examined whether this agent could cooperate with weekly dose of RMC-6272 followed by triweekly doses of a-PD-1 for 28 days (Fig. 5a). Of note, tumors in our autochthonous MYC-driven HCC mouse model do not respond to a-PD-1 therapy as shown in Fig. $5 b-5 c$, similar to clinical reports using single immune checkpoint inhibitors in $\mathrm{HCC}^{56-58}$. Treatment with RMC-6272 alone or in combination with a-PD-1 markedly reduced the number of tumor nodules and the percent liver weight/body weight compared to controls or single agent $\alpha-P D-1$ treatment (Fig. 5b-5c). RMC-6272 treatment effects at Day 7 were consistent with those observed in the single dose study (Fig.

3f-3k and Fig. 5d-5f). Interestingly, in the 28-day efficacy study, we observed on-treatment tumor volume increase in theRMC-6272-treated mice, with 8 of 11 mice showing tumor volume increase from baseline over time as assessed by tumor volume change over time calculated from 2D MRI images, and tumor area change over time calculated from 3D reconstructions of MRI images (Fig. 5e and Sup. Fig. 11). In contrast, only 1 of $11(9 \%)$ mice treated with the combination therapy showed a slight tumor volume increase by week 4, indicating a combinatorial impact on treatment durability. Another indicator of a clear 
681 combinatorial effect of RMC-6272 with a-PD-1 was the increased tumor regression compared to either

682 RMC-6272 or a-PD-1 alone (38\%, 18\%, and 0\%, respectively, Fig. 5f, and Sup. Table 2). Furthermore, 683 using tumor volume doubling as a surrogate for disease progression, combination of RMC-6272 with a684 PD-1 significantly improved progression-free survival compared to either agent alone (Fig. 5g). Long685 term treatment with RMC-6272 alone or in combination with a-PD-1 was not associated with body weight 686 loss or hepatotoxicity (Sup. Fig. 12). Thus, combining a selective bi-steric mTORC1 inhibitor with $\alpha$-PD6871 treatment induced sustained tumor regressions in a MYC-driven HCC model.

688

The combination of RMC-6272 with $\alpha-P D-1$ increased T and NK cell recruitment and the release of perforins and granzymes:

691 We next examined potential mechanisms of the combinatorial effects on tumor suppression described above. Immune checkpoint therapy is associated with immune activation including $\mathrm{T}$ cell and NK cell degranulation and the release of perforins and granzymes to control tumor growth ${ }^{59,60}$. We found that single agent RMC-6272 and more so, RMC-6272 and $\alpha-P D-1$, increase intratumoral abundance of CD4+ and NKp46+ (NK cell marker) immune cells in MYC-driven tumors compared to vehicle- or a-PD-1-treated tumors suggesting T and NK cell activation (Fig. 5h-5i). RMC-6272 with a-PD-1 increased NKG7 levels compared to a-PD-1 or RMC-6272 alone even though RMC-6272 alone enhanced recruitment of CD4+ and NKp46+ cells, consistent with increased degranulation (Fig. 5h-5i). RMC-6272 with a-PD-1, more than either agent alone, increased levels of perforins and granzyme B (Fig. 5h-5i). Thus, RMC-6272 with a-PD-1 increased recruitment of CD4+ cells, immune cell degranulation, and release of perforins and 701 granzyme B in MYC-driven tumors, relative to single agent RMC-6272, and consistent with the enhanced 702 anti-tumor effects resulting from the combination therapy. 


\section{Discussion:}

707 The mTOR pathway is key to regulating the protein translation of MYC. MYC is global a driver of cancer 708 cell growth and immune evasion, but remains to be therapeutically targeted ${ }^{8}$. The inhibition of the mTOR 709 pathway, in particular mTORC1 and leading to downstream activation of 4EBP1, and the consequent 710 inhibition of cap-dependent translation, has therapeutic potential in MYC-driven tumors ${ }^{15}$. First generation 711 mTORC1 selective inhibitors such as Rapalogs are unable to reactivate 4EBP1, have shown limited 712 clinical efficacy, and also appear to have dose limiting hepatic and immune toxicity $1,16,31,33$; second 713 generation nonselective mTOR inhibitors of both mTORC1 and mTORC2 have had limited clinical 714 success perhaps due to a narrow therapeutic index ${ }^{19-21}$. We speculated that an mTORC1 selective inhibitor that engaged 4EBP1 would be more effective at suppressing MYC and have less nonspecific toxicity. Here we demonstrate that bi-steric selective mTORC1 inhibitors that activate 4EBP1 result in marked suppression of MYC protein levels, and drive anti-tumor activity, including regressions, in both a transgenic mouse model of MYC-driven HCC and in human PDX models of MYC-amplified HCC, colorectal cancer, head and neck cancer, and ovarian cancer (Fig. 6 (1)). Furthermore, the present data suggest that bi-steric mTORC1-selective inhibitors are not immune suppressive or hepatotoxic, but rather, elicit robust anti-cancer immunity and cooperate with immune checkpoint therapy (Fig. 6 (2)).

mTORC1 inhibition activates 4EBP1, thereby decreasing MYC protein expression and producing an antitumor effect ${ }^{1,10-13}$. Using representative bi-steric mTORC1-selective inhibitors to conduct preclinical studies in our MYC-driven HCC mouse model, we found that pharmacological inhibition of mTORC1 is 726 very similar to genetic MYC inactivation. Our results suggest a model whereby mTORC1 and p4EBP1 727 play a key role in regulating MYC protein expression serving as a nodal point for the maintenance of a cancer phenotype (see Figs. 2-3). We also found that high EIF4EBP1 expression and high MYC signaling are associated with poorer survival in human HCC patients when compared with patients with low 
732 undergoing clinical testing (NCT04774952), has anti-tumor activity against a panel of PDX tumors with 733 MYC amplification.

Selective mTORC1 inhibition elicits immune activation and cooperates with $\alpha$-PD-1 to induce tumor regression in a transgenic mouse model of MYC-driven HCC. We propose this immune activation occurs through two mechanisms:

1) Depletion of MYC in vivo may result in the inhibition of cell intrinsic programs that promote cell growth including cancer cell proliferation ${ }^{61}$ and cell cycle progression ${ }^{62}$, and 2) in the induction of programs that enhance cell death including apoptosis ${ }^{35}$ and decreased metabolism and ribosomal biosynthesis ${ }^{40}$. Dying cancer cells release damage associated molecular patterns (DAMPs) which promote innate anti-tumor immunity and antigen presentation by antigen-presenting cells (APCs) which prime T cells inducing their recruitment to the tumor ${ }^{63,64}$. Moreover, MYC depletion in vivo by selective mTORC1 inhibitors may reduce the expression of immune checkpoint receptors (ICRs $)^{37}$ on cancer cells further enhancing tumor recruitment of adaptive immune cells.

Here, we showed that inhibiting mTORC1 depletes MYC eliciting immune activation as measured by GSEA and GO Term enrichment analyses of RNA-seq data, CIBERSORT ${ }^{46}$ and TIMER2.047, and CODEX ${ }^{55,65}$. This includes the recruitment and activation of CD4+ T cells ${ }^{66}$, maturation and activation of NK cells ${ }^{67}$, and prevention of M2 macrophage polarization ${ }^{8,44}$ (Fig. 4). In contrast, nonselective mTOR 752 inhibition has been shown to cause $\mathrm{T}$ cell anergy ${ }^{25,68}$, decrease NK cell proliferation ${ }^{26}$, enhance $\mathrm{T}_{\text {reg }}$ 753 differentiation and expansion ${ }^{28,29}$, and reduce expression of granzyme $\mathrm{B}$ and interferon gamma by 754 decreasing TCR/mTORC1/IL-2R signaling ${ }^{23,24}$. Second, nonselective mTOR inhibitors reduce AKT 755 activation in both $\mathrm{T}$ and NK cells, which would prevent mTORC1 activation and thereby immune cell 756 proliferation ${ }^{30,69}$. Thus, bi-steric mTORC1-selective inhibitors most likely activate immune responses both 757 by reducing MYC protein expression resulting in reduced ICR expression and DAMP release and by not inhibiting AKT, contributing to enhanced immunity. 
760 Finally, we found that RMC-6272 cooperates with immune checkpoint inhibitors to induce tumor 761 regression in the aforementioned mouse model of MYC-driven HCC. Earlier generations of mTOR 762 inhibitors have been shown to be immunosuppressive ${ }^{18,22,31,23-30}$, raising the concern that bi-steric mTOR 763 inhibitors may interfere with immune therapies including immune checkpoint inhibitors. Current approved 764 tyrosine kinase inhibitors for HCC, including sorafenib, have shown a limited ability to induce immune 765 activation ${ }^{70-73}$ and to synergize with immune checkpoint inhibitors in HCC $^{74-77}$. Here, we first reported 766 that RMC-6272 alone induces immune activation in MYC-driven HCC tumors performing significantly 767 better than sorafenib (Fig. 4). Second, we showed that RMC-6272 transformed the tumor immune 768 microenvironment and synergized with a-PD-1 to elicit sustained tumor regression, increased T and NK 769 cell recruitment, and the release of perforins and granzymes (Fig. 5). Selective mTORC1 inhibition via 770 RMC-6272 most likely sensitizes MYC-driven tumors to a-PD-1 immunotherapy by overcoming MYC771 driven immune evasion ${ }^{8,78}$. Therefore, bi-steric mTORC1-selective inhibitors have the potential to 772 clinically improve immune checkpoint inhibitor responses in patients with MYC-overexpressing cancers. 773 In sum, our preclinical results support the conclusion that targeting mTORC1 has therapeutic potential in 774 the treatment of MYC-driven cancers alone and in combination with immune checkpoint inhibition, 775 concepts that inform the clinical testing of RMC-5552 (NCT04774952). 


\section{Acknowledgments}

We thank the Stanford Division of Oncology, and the Stanford Department of Medicine for scientific advice. We thank Delaney Sullivan for helping us running signscore and running CIBERSORT. We thank Pauline Chu in the Department of Pathology at Stanford University for helping us with tissue sectioning for immunohistochemical studies. We thank the Translational Applications Service Center (TASC) and especially Joanna Liliental and Shailja Patel for their help with Nanoimmunoassay. We thank the Stanford Cell Sciences Imaging Facility (CSIF) and Anum Khan for sample processing for CODEX. We also thank the Stanford Animal Diagnostics Laboratory and Animal Histology Service for analyses of serum samples and generation of frozen slides, respectively. We thank Revolution Medicines' colleagues Elsa Quintana,

Pete Wildes, Bianca Lee, Jingjing Jiang for key scientific input and review, as well as Ethan Ahler and studies. Figures were made using Biorender and the Qlucore software was used to analyze and generate figures from RNA-seq data.

\section{Funding Sources}

This work was supported by a grant from Revolution Medicines to DWF.

\section{Conflict of Interest}

JAMS, MS, YCY, JWE, GLB, and AG are employees and shareholders of Revolution Medicines. DWF, 798

\section{Authors' contributions}

800 WDMF, YCY, JAMS, MS, and DWF conceived the study. MS, WDMF, and YCY developed the experimental methodology. WDMF, YCY, IL, JP, LY, and JWE conducted experiments. WDMF performed experiments, analyzed data and generated figures. WDMF wrote the manuscript. JAMS, MS, GLB, AG, 
bioRxiv preprint doi: https://doi.org/10.1101/2022.02.04.478208; this version posted February 5, 2022. The copyright holder for this preprint (which was not certified by peer review) is the author/funder, who has granted bioRxiv a license to display the preprint in perpetuity. It is made available under aCC-BY-ND 4.0 International license.

803 DWF, WDMF and YCY edited the manuscript. JAMS and DWF provided administrative, technical, and

804 material support. JAMS, MS and DWF supervised the study. All authors read and approved the final 805 manuscript. 


\section{Figure Legends:}

Figure 1. Bi-steric mTORC1-selective inhibitors reduce the growth of MYC-driven HCC cells and

MYC protein levels. a, Graphical depiction of the study showing overactivation of mTOR signaling in cancer cells (left), the effect of current generation of mTOR inhibitors in mTORC1 and mTORC2 signaling as well as in anti-cancer immune responses (middle) and, the proposed effect of mTORC1 selective inhibitors in $\mathrm{mTORC} 1$ and mTORC2 signaling as well as in tumor growth and anti-cancer immunity tested in this study. $\mathbf{b}$, Structure of the rapamycin-like core (left) which is part of the bi-steric selective mTORC1 inhibitors-RMC-4627, RMC-6272, and RMC-5552. Structures of the ATP competitive inhibitors used for each inhibitor (right). Table depicts the selectivity of each these inhibitors for mTORC1 relative to mTORC2. c, relative transcript levels of the human MYC transgene (hMYC) and endogenous mouse MYC (mMYC), d, representative western blot and relative levels of human MYC (hMYC) and alpha tubulin ( $\alpha$-Tubulin) protein in EC4 treated cells depicted as percent relative to vehicle, e, Relative mRNA levels of four MYC-regulated target genes including Apex1, Srm, Cct3, and S6K (T389), and total p70 S6 Kinase, mTORC2 factors pAKT (S473) and total AKT, and loading control beta actin ( $\beta$-actin) from EC4 MYC-driven HCC cells treated with doxycycline at $20 \mathrm{ng} / \mathrm{ml}$ or with mTORC1 selective inhibitors RMC-4627 and RMC-6272 at $100 \mathrm{nM}$ for 48 hours. Table in the bottom depicts the percent inhibition of pAKT, p4EBP1, and pS6 for each treatment group. g, Relative growth

RMC-6272. Numbers under western blot represents relative protein levels compared to vehicle-treated cells which were set at $100 \%$. Error bars represent SEM. Significance was taken at $\mathrm{P}<0.05\left(^{*}\right), P<0.01$ $\left.\left({ }^{* *}\right), \mathrm{P}<0.005{ }^{(* *}\right)$, and $\left.\mathrm{P}<0.001{ }^{(* * *}\right)$. ns $=$ not significant. 
833 (blue) in MYC signature (MYC ${ }^{\text {Sig }}$ ) high versus low human HCC tumors (TCPA LIHC). Names of proteins

834 which are specific to the mTORC1 pathway are highlighted in red. b, Overall survival of human HCC

835 patients (TCGA LIHC) after stratifying them into those expressing MYC ${ }^{\text {Sig }}$ high $\left(\mathrm{MYC}^{\mathrm{Sig}}+\right)$ and low

836 (MYC $\left.{ }^{\text {Sig_- }}\right)$ and expressing high or low levels of EIF4EBP1 $\left(\mathrm{n}=20\right.$ for MYC ${ }^{\mathrm{Sig}}+/ 4 \mathrm{EBP} 1+$ and MYC Sig_

837 /4EBP1-; and, n=14 for MYC Sig +/4EBP1- and MYC Sig_/4EBP1+). c, Representative western blot-like

838 images generated from size separation nanoimmunoassay (NIA), d, representative

839 immunohistochemistry (IHC) images, e, representative NIA western blot-like images, and $\mathbf{f}$,

840 quantification of the different 4EBP1 phosphorylation isoforms from charge separation NIA. g-j,

841 Enrichment scores (ES) of $\mathbf{g}$, hallmarks of MYC targets geneset, $\mathbf{h}$, hallmarks of mTORC1 signaling

842 geneset, i, hallmarks of PI3K, AKT, mTOR signaling geneset, and j, activated MAPK activity geneset;

843 and $\mathbf{k}-\mathbf{n}$, quantification of $\mathbf{k}$, hMYC, I, p4EBP1, $\mathbf{m}, \mathrm{pS6RP}, \mathbf{n}, \mathrm{pAKT}$ over $\beta$-macroglobulin protein from

844 NIA analysis, from HCC tumors of Lap-tTA/Tet-O-MYC mice treated for 7 days with a vehicle control

845 (5/5/90 Transcutol/Solutol HS 15/Water) injected intraperitoneally once weekly, doxycycline at 100

$846 \mathrm{ug} / \mathrm{ml}$ in water (MYC Off), sorafenib at $30 \mathrm{mg} / \mathrm{kg}$ given via oral gavage once daily for a week or two bi-

847 steric mTORC1-selective inhibitors RMC-4627 and RMC-6272 at $10 \mathrm{mg} / \mathrm{kg}$ intraperitoneally once

848 weekly. At day 7, mice were treated with one more dose of their respective treatment and euthanized at

$8494,12,24,48$, or 72 hours after. In panels $\mathbf{c}$ and $\mathbf{d}$, the number of "p"s presiding the protein name

850 corresponds to the relative phosphorylation levels. Thus, ppp-4EBP1 is more phosphorylated than the

851 p-4EBP1 isoform; Up $=$ unphosphorylated. Error bars represent SEM. Significance was taken at $\mathrm{P}<0.05$

$\left.852\left(^{*}\right), \mathrm{P}<0.01\left(^{* *}\right), \mathrm{P}<0.005\left(^{* * *}\right), \mathrm{P}<0.001{ }^{* * * *}\right) . \mathrm{ns}=$ not significant. Scale bar in panel $\mathbf{d}$ is $100 \mu \mathrm{m}$.

Figure 3. Bi-steric mTORC1 inhibitors induce regression of human and mouse MYC-driven HCC

855 tumors. a-d, Tumor volume over time of four HCC patient-derived xenografts (PDXs) with different 856 copy number variants (CNV) of MYC ranging from low (left) to high (right) treated with a vehicle control 857 (5/5/90 Transcutol/Solutol HS 15/Water) or RMC-5552 given intraperitoneally at 3 or $10 \mathrm{mg} / \mathrm{kg} / \mathrm{week}$. e, 858 Graphical depiction of the methods. Lap-tTA/Tet-O-MYC mice were taken off doxycycline (DOX) to 859 induce hMYC expression and induce hepatocellular carcinoma initiation. Three weeks after, mice were 
MRI imaged to assess tumor volumes before enrolling them in different treatment groups which include vehicle control (5/5/90 Transcutol/Solutol HS 15/Water) injected intraperitoneally once weekly, doxycycline given at $100 \mu \mathrm{g} / \mathrm{ml}$ in water (MYC Off), sorafenib at $30 \mathrm{mg} / \mathrm{kg}$ given via daily oral gavage and two selective mTORC1 inhibitors RMC-4627 and RMC-6272 at $10 \mathrm{mg} / \mathrm{kg}$ weekly. f, Number of liver tumor nodules, g, Representative MRI images (left) and 3D HCC tumors (right), h, tumor volume change relative to Day 0 for each treatment group, $\mathbf{i}$, tumor area change over time depicted as a percent and calculated from 3D HCC tumors before (Day 0) and after (Day 7) treatment, j, liver weight over body weight, and $\mathbf{k}$, incidence of tumor regression of Lap-tTA/Tet-O-MYC mice treated with a vehicle control, doxycycline, sorafenib, RMC-4627, or RMC-6272 for 7 days. Error bars represent minimum and maximum whiskers. Tumor volumes at the end of study was compared between control and treatment groups by two-way ANOVA Dunnet's multiple comparison test. Significance was taken at $\mathrm{P}<0.05\left(^{*}\right), \mathrm{P}<0.01\left(^{* *}\right)$, and $\mathrm{P}<0.005\left(^{* * *}\right)$. ns $=$ not significant.

Figure 4. RMC-6272 induces immune activation in MYC-driven HCC tumors. a, Heat map of genes in the Immune System Process geneset, b, Enrichment scores over time from the Immune System Process geneset, c, GSEA plots from the Immune System Process geneset over time, and d-f, GO terms enrichment analysis from transcriptome data of HCC tumors of Lap-tTA/Tet-O-MYC mice treated with a vehicle control (5/5/90 Transcutol/Solutol HS 15/Water) injected intraperitoneally once weekly, doxycycline at $100 \mathrm{ug} / \mathrm{ml}$ in water (MYC Off), sorafenib at $30 \mathrm{mg} / \mathrm{kg}$ given via daily oral gavage, or the bi-steric mTORC1-selective inhibitor RMC-6272 injected intraperitoneally at $10 \mathrm{mg} / \mathrm{kg}$ once weekly. Mice were euthanized for sample collections at the following time points after a final dose on Day 7 , whereas for the bi-steric inhibitors, a second dose was given followed by euthanasia and sample collections at $\mathbf{d}, 24, \mathbf{e}, 48$, and $\mathbf{f}, 72$ hours post-dosing. $\mathbf{g}$, Heat map of the relative abundance of different immune populations in HCC tumors of mice treated with vehicle, doxycycline (MYC Off), RMC4627, RMC-6272, or sorafenib using CIBERSORT and TIMER2.0. The sum of the abundances of all the different immune subsets per sample equals one. $\mathbf{h}$ and $\mathbf{i}$, Representative $\mathbf{h}$, high magnification, and $\mathbf{i}$, low magnification images from CODEX analysis, and $\mathbf{j}$, quantification of protein levels of 5 
markers (Ki67, CD4, CD8a, CD11c, and CD19) in HCC tumors from Lap-tTA/Tet-O-MYC mice treated

for 7 days with a vehicle control, doxycycline (MYC Off), or RMC-6272. White arrow heads, in panel h,

depict CD4+ immune cells that are present in areas with Ki67 low/- cells. $\mathbf{k}$, Correlation analyses of

abundance of CD4+ cells relative to Ki67+ cells quantified from CODEX data from HCC tumors of Lap-

tTA/Tet-O-MYC mice treated with a vehicle control (black), RMC-6272 (light blue) or doxycycline (MYC

Off, orange). Numbers next to legend correspond to the tumor volume change at Day 7 post treatment depicted as a percent relative to Day 0. Error bars represent SEM. Scale bar in panel $\mathbf{h}$ is $100 \mu \mathrm{m}$ and in panel $\mathbf{i}$ it si $1 \mathrm{~mm}$.

Figure 5. RMC-6272 in combination with a-PD-1 induces immune degranulation and sustained over body weight at day $28, \mathbf{d}$, representative $\mathrm{MRI}$ images over time, $\mathbf{e}$, tumor volume change over time relative to Day 0 for each treatment group, $\mathbf{f}$, incidence of tumor regression over time, $\mathbf{g}$, progression-

free survival, and $\mathbf{h}$, representative immunohistochemistry images, and $\mathbf{i}$, quantification of CD4, NKp46, NKG7, perforins, and granzyme B (Granz. B) in HCC tumors from Lap-tTA/Tet-O-MYC treated for 28 days with a vehicle control (5/5/90 Transcutol/Solutol HS 15/Water), RMC-6272 at 10 mg/kg injected intraperitoneally once weekly, a-PD-1 injected intraperitoneally at 300 ug triweekly, or a combination of each treatment group. Log rank (Mantel-Cox) test was used for Kaplan-Meier survival analyses to compare indicated groups. Significance was taken at $\mathrm{P}<0.05\left(^{*}\right), \mathrm{P}<0.01\left({ }^{* *}\right)$, and $\mathrm{P}<0.001\left({ }^{* * *}\right)$. ns $=$ RMC-6272 and $\alpha-P D-1$. Error bars represent minimum and maximum whiskers. The numbers in panel $\mathbf{i}$ represent the relative number of positive cells per field of view for different areas of tumors from mice in not significant. Scale bar in panel $\mathbf{h}$ is $50 \mu \mathrm{m}$. 
Figure 6. Model of anti-tumor immunity induction by the therapeutic combination of bi-steric mTORC1 inhibitors with immunotherapy. MYC-driven cancers have high p4EBP and pS6K levels which increase MYC translation inducing tumor growth and immune evasion characterized by

decreased abundance of CD4+ T cells and NKp46+ NK cells; and low levels of perforins and granzyme regression and immune activation enhancing recruitment of CD4+ T cells and NKp46+ NK cells. degranulation, and the release of perforins and granzyme B (right, (2)).

\section{Supplemental Figure Legends:}

\section{Supplemental Figure 1. Low MYC signaling and EIF4EBP1 levels in human HCC tumors are}

predictive of worse survival. a-b, Kaplan Meier survival plots of $\mathbf{a}$, progression-free survival and $\mathbf{b}$, disease-free survival of human HCC patients from TCGA LIHC after stratifying them into those expressing high and low MYC signature (MYC $\left.{ }^{\mathrm{Sig}}\right)$, using the Hallmarks of MYC targets geneset and signscore, and expressing high or low EIF4EBP1 levels. Log rank (Mantel-Cox) test was used for Kaplan-Meier survival analyses to compare indicated groups. Significance was taken at $\mathrm{P}<0.05\left(^{*}\right)$ and $\mathrm{P}<0.005\left(^{* * *}\right) . \mathrm{ns}=$ not significant

\section{Supplemental Figure 2. The bi-steric mTORC1-selective inhibitor RMC-6272 potently reduces}


941 doxycycline at $100 \mu \mathrm{g} / \mathrm{ml}$ in water (MYC Off), sorafenib at $30 \mathrm{mg} / \mathrm{kg} /$ day given via oral gavage, or two

942 bi-steric mTORC1- selective inhibitors RMC-4627 and RMC-6272 at $10 \mathrm{mg} / \mathrm{kg}$ injected intraperitoneally

943 once weekly. At day 7, mice were treated with one more dose of their respective treatment and

944 euthanize 24 hours after. Error bars represent minimum and maximum whiskers for panels $\mathbf{a}$ and $\mathbf{b}$ and

945 SEM for panel d. Significance was taken at $\mathrm{P}<0.05\left({ }^{*}\right), \mathrm{P}<0.01\left(^{* \star}\right), \mathrm{P}<0.005\left(^{* \star *}\right)$, and $\mathrm{P}<0.001\left(^{* \star * *}\right)$.

946 ns = not significant.

\section{Supplemental Figure 3. RMC-6272 reduces the expression of mTORC1- and MYC-target genes}

without affecting expression of genes regulated by mTORC2 or MAPK. a, Heat maps of genes and

b, GSEA plots of the following genesets: hallmarks of MYC targets, hallmarks of mTORC1 signaling, euthanize 24 hours after.

\section{Supplemental Figure 4. Effect of RMC-6272 on the expression of genes regulated by oncogenic}

signaling pathways common to HCC. a-h, Heat maps (top) and GSEA plots (bottom) of the following

genesets: a, hallmarks of MYC targets, b, canonical WNT signaling pathway, c, neoplastic

transformation by CCND1 (WNT) and MYC, d, RAS signaling pathway, e, HRAS oncogenic signature,

f, KRAS signaling downregulated genes, $\mathbf{g}$, reactome signaling by BRAF and RAF fusions, and $\mathbf{h}, \mathrm{PI} 3 \mathrm{~K}$ and AKT signaling pathway from RNA-seq data of HCC tumors in Lap-tTA/Tet-O-MYC mice treated for 7 days with a vehicle control (5/5/90 Transcutol/Solutol HS 15/Water), doxycycline at $100 \mu \mathrm{g} / \mathrm{ml}$ in water (MYC Off), Sorafenib at $30 \mathrm{mg} / \mathrm{kg} /$ day given via oral gavage or the selective mTORC1 inhibitors 
968 with one more dose of their respective treatment and euthanize 24 hours after. ES $=$ enrichment

969 scores.

Supplemental Figure 5. RMC-6272 induces a durable decrease of p4EBP1, pS6RP, and MYC protein levels compared to RMC-4627. a-d, Quantification of a, hMYC, b, p4EBP1, and c, pS6RP 973 protein levels as detected by immunohistochemistry. Lap-tTA/Tet-O-MYC mice were treated for 7 days with a vehicle control (5/5/90 Transcutol/Solutol HS 15/Water) injected intraperitoneally once weekly, doxycycline at $100 \mu \mathrm{g} / \mathrm{ml}$ in water (MYC Off), sorafenib at $30 \mathrm{mg} / \mathrm{kg} /$ day given via oral gavage, or two bi-steric mTORC-selective inhibitors RMC-4627 and RMC-6272 at $10 \mathrm{mg} / \mathrm{kg}$ injected intraperitoneally once weekly. Mice were euthanized for tumor collection at the following time points after a final dose on Day 7: 4, 12, 24, 47, and 72 hours. Error bars represent SEM. Significance was taken at $P<0.01\left({ }^{* *}\right)$, and $\mathrm{P}<0.005\left(^{* * *}\right) . \mathrm{ns}=$ not significant. two-way ANOVA Dunnet's multiple comparison test. Significance was taken at $\mathrm{P}<0.05\left({ }^{\star}\right), \mathrm{P}<0.01\left(^{* *}\right)$, and $\mathrm{P}<0.005\left(^{* * *}\right)$, ns $=$ not significant.

\section{Supplemental Figure 7. RMC-6272 induces regression of high burden MYC-driven tumors.}

990 Representative a, MRI images, b, 3D HCC tumors, and $\mathbf{c}-\mathbf{g}$, tumor volume over time of HCC tumors in 991 Lap-tTA/Tet-O-MYC mice before (Day 0) and after (Day 7) treatment with c, a vehicle control (5/5/90 992 Transcutol/Solutol HS 15/Water) injected intraperitoneally once weekly, d, doxycycline at $100 \mu \mathrm{g} / \mathrm{ml}$ in water (MYC Off), two pre-clinical bi-steric selective mTORC1 inhibitors e, RMC-4627 and f, RMC-6272 


\section{Supplemental Figure 8. Bi-steric mTORC1 inhibitors do not induce body weight loss or liver}

997 toxicity in MYC-driven HCC model. a, body weight change at Day 7 depicted as a percent over initial 998 weight at Day 0 (before treatment), and $\mathbf{b}$, levels of circulating liver enzymes alanine aminotransferase 999 (ALT), aspartate transaminase (AST), alkaline phosphatase (Alk. Phos.), bilirubin, gamma-glutamyl 1000 transferase (GGT); and, c, cholesterol, d, triglycerides, e, sodium, f, high-density lipoprotein (HDL), $\mathbf{g}$, 1001 low-density lipoprotein (LDL), h, chloride, i, glucose, j, albumin, k, creatinine, I, blood urea nitrogen 1002 (BUN), $\mathbf{m}$, calcium, $\mathbf{n}$, globulin, o, potassium $\mathbf{p}, \mathrm{CO}_{2}$, and $\mathbf{q}$, total protein levels from serum of Lap1003 tTA/Tet-O-MYC mice treated with a vehicle control (5/5/90 Transcutol/Solutol HS 15/Water) injected 1004 intraperitoneally once weekly, doxycycline, sorafenib $30 \mathrm{mg} / \mathrm{kg}$ daily orally, once weekly intraperitoneal 1005 injection of RMC-4627, or RMC-6272 for 7 days. Gray squares depict the range of normal values for specific factors in wild-type mice. Error bars represent minimum and maximum whiskers. Significance was taken at $\mathrm{P}<0.05\left({ }^{*}\right), \mathrm{P}<0.01\left(^{* *}\right), \mathrm{P}<0.005\left(^{* * *}\right)$, and $\mathrm{P}<0.001\left(^{* * *}\right)$. ns $=$ not significant.

Supplemental Figure 9. Bi-steric mTORC1 inhibitors are not associated with hyposplenism in MYC-driven HCC model. Spleen sizes of Lap-tTA/Tet-O-MYC mice treated for 7 days with a vehicle control (5/5/90 Transcutol/Solutol HS 15/Water) injected intraperitoneally once weekly, RMC-4627 or RMC-6272 at $10 \mathrm{mg} / \mathrm{kg}$ injected intraperitoneally once weekly, or sorafenib at $30 \mathrm{mg} / \mathrm{kg} /$ daily via oral 1013 gavage. Error bars represent SEM. Significance was taken at $\mathrm{P}<0.05\left(^{*}\right)$.

Supplemental Figure 10. MYC signature and EIF4EBP1 downregulation is predictive of immune

reactivation. Heat map depicting relative abundance of different immune populations in MYC ${ }^{\mathrm{Sig}}$ and 1017 EIF4EBP1 high versus low HCC tumors (TCGA LIHC) using CIBERSORT.

Supplemental Figure 11. RMC-6272 in combination with $\alpha-P D-1$ drives tumor regression in a 
1022 tTA/Tet-O-MYC mice before treatment (Day 0) and after weekly treatments for 28 days with c, a vehicle 1023 control (5/5/90 Transcutol/Solutol HS 15/Water) injected intraperitoneally once weekly, d, a-PD-1

1024 injected intraperitoneally at $300 \mu \mathrm{g}$ triweekly, e, RMC-6272 injected intraperitoneally at $10 \mathrm{mg} / \mathrm{kg} / \mathrm{week}$, 1025 or $\mathbf{f}$, a combination of RMC-6272 with $\alpha-P D-1$. Scale bar in panel $\mathbf{g}$ is $10 \mathrm{~mm}$.

\section{Supplemental Figure 12. Effects of RMC-6272 in combination with $\alpha-P D-1$ in levels of several}

1028 circulating factors. a, Levels of circulating liver enzymes: alanine aminotransferase (ALT), aspartate

1029 transaminase (AST), alkaline phosphatase (Alk. Phos.), bilirubin, and gamma-glutamyl transferase 1030 (GGT) from Lap-tTA/Tet-O-MYC mice treated with vehicle, RMC-6272, $\alpha$-PD-1, or RMC-6272 with $\alpha$ 1031 PD-1 for 28 days. b, Body weight change over time depicted as a percent over initial weight at Day 0 1032 (before treatment). c-q, Levels of circulating c, cholesterol, $\mathbf{d}$, triglycerides, e, sodium, f, potassium, $\mathbf{g}$, 1033 high-density lipoprotein (HDL), h, low-density lipoprotein (LDL), i, chloride, j, CO, , k, glucose, I, 1034 albumin, $\mathbf{m}$, creatinine, $\mathbf{n}$, total protein, $\mathbf{0}$, blood urea nitrogen (BUN), $\mathbf{p}$, calcium, and $\mathbf{q}$, globulin levels 1035 from serum of Lap-tTA/Tet-O-MYC mice before treatment (Day 0) and after weekly treatments for 28 1036 days with a vehicle control (5/5/90 Transcutol/Solutol HS 15/Water) injected intraperitoneally once 1037 weekly, a-PD-1 injected intraperitoneally at $300 \mu \mathrm{g}$ triweekly, RMC-6272 injected intraperitoneally at 10 $1038 \mathrm{mg} / \mathrm{kg} /$ week, or a combination of RMC-6272 with $\alpha-P D-1$. Gray squares depict the range of normal 1039 values for specific factors in wild-type mice. Error bars represent minimum and maximum whiskers. 1040 Significance was taken at $\mathrm{P}<0.05\left(^{*}\right)$ and $\mathrm{P}<0.01\left(^{* *}\right) . \mathrm{ns}=$ not significant. 


\section{References:}

1044 1. Liu, P. et al. A functional mammalian target of rapamycin complex 1 signaling is indispensable 1045 for c-Myc-driven hepatocarcinogenesis. Hepatology (2017). doi:10.1002/hep.29183

$10462 . \quad$ Forbes, S. A. et al. COSMIC: Mining complete cancer genomes in the catalogue of somatic mutations in cancer. Nucleic Acids Res. (2011). doi:10.1093/nar/gkq929

3. Mossmann, D., Park, S. \& Hall, M. N. mTOR signalling and cellular metabolism are mutual determinants in cancer. Nature Reviews Cancer (2018). doi:10.1038/s41568-018-0074-8

5. Pirazzoli, V. et al. Acquired resistance of EGFR-mutant lung Adenocarcinomas to Afatinib plus

6. Kawabata, S. et al. Rapamycin Prevents the Development and Progression of Mutant Epidermal (2014). doi:10.1016/j.celrep.2014.05.039

7. Elkabets, M. et al. MTORC1 inhibition is required for sensitivity to PI3K p110a inhibitors in

8. Dhanasekaran, R. et al. The MYC oncogene - the grand orchestrator of cancer growth and immune evasion. Nat. Rev. Clin. Oncol. 2021 1-14 (2021). doi:10.1038/s41571-021-00549-2

9. Ally, A. et al. Comprehensive and Integrative Genomic Characterization of Hepatocellular Carcinoma. Cell 169, 1327-1341.e23 (2017).

10. Thoma, C., Bergamini, G., Galy, B., Hundsdoerfer, P. \& Hentze, M. W. Enhancement of IRESmediated translation of the c-myc and BiP mRNAs by the poly $(A)$ tail is independent of intact elF4G and PABP. Mol. Cell (2004). doi:10.1016/j.molcel.2004.08.021

11. Wall, M. et al. Translational control of c-MYC by rapamycin promotes terminal myeloid 
12. Csibi, A. et al. The mTORC1/S6K1 pathway regulates glutamine metabolism through the eif4bdependent control of c-Myc translation. Curr. Biol. (2014). doi:10.1016/j.cub.2014.08.007

13. Babcock, J. T. et al. Mammalian Target of Rapamycin Complex 1 (mTORC1) enhances bortezomib-induced death in Tuberous Sclerosis Complex (TSC)-null cells by a c-MYCdependent induction of the unfolded protein response. J. Biol. Chem. (2013). doi:10.1074/jbc.M112.431056

14. Liu, G. Y. \& Sabatini, D. M. mTOR at the nexus of nutrition, growth, ageing and disease. Nature Reviews Molecular Cell Biology (2020). doi:10.1038/s41580-019-0199-y

15. Pourdehnad, M. et al. Myc and mTOR converge on a common node in protein synthesis control that confers synthetic lethality in Myc-driven cancers. Proc. Natl. Acad. Sci. U. S. A. (2013).

16. Xu, Z. et al. The mTORC2-Akt1 Cascade Is Crucial for c-Myc to Promote Hepatocarcinogenesis

17. Fruman, D. A. et al. The PI3K Pathway in Human Disease. Cell 170, 605-635 (2017).

18. Ferreiro, A. O. et al. Everolimus-based immunosuppression in patients with hepatocellular carcinoma at high risk of recurrence after liver transplantation: A case series. Transplant. Proc. (2014). doi:10.1016/j.transproceed.2014.08.045

19. Hua, H. et al. Targeting mTOR for cancer therapy. Journal of Hematology and Oncology (2019).

21. Ghobrial, I. M. et al. TAK-228 (formerly MLN0128), an investigational oral dual TORC1/2 inhibitor: A phase I dose escalation study in patients with relapsed or refractory multiple myeloma, non-Hodgkin lymphoma, or Waldenström's macroglobulinemia. Am. J. Hematol. 91, 
1096 22. Umemura, A. et al. Liver damage, inflammation, and enhanced tumorigenesis after persistent mTORC1 inhibition. Cell Metab. (2014). doi:10.1016/j.cmet.2014.05.001

23. Ferrer, I. R., Araki, K. \& Ford, M. L. Paradoxical aspects of rapamycin immunobiology in transplantation. American Journal of Transplantation (2011). doi:10.1111/j.1600-

24. Bak, S. et al. Selective Effects of mTOR Inhibitor Sirolimus on Naïve and CMV-Specific T Cells doi:10.3389/fimmu.2018.02953

25. Araki, K., Youngblood, B. \& Ahmed, R. The role of mTOR in memory CD8+ T-cell differentiation.

26. Viel, S. et al. TGF- $\beta$ inhibits the activation and functions of NK cells by repressing the mTOR

27. Pollizzi, K. N. et al. mTORC1 and mTORC2 selectively regulate CD8+ T cell differentiation. J.

28. Battaglia, M. et al. Rapamycin Promotes Expansion of Functional CD4 + CD25 + FOXP3 +

32. Lee, B. J. et al. Selective inhibitors of mTORC1 activate 4EBP1 and suppress tumor growth. Nat. Regulatory T Cells of Both Healthy Subjects and Type 1 Diabetic Patients . J. Immunol. (2006). doi:10.4049/jimmunol.177.12.8338

29. Delgoffe, G. M. et al. The mTOR Kinase Differentially Regulates Effector and Regulatory T Cell Lineage Commitment. Immunity (2009). doi:10.1016/j.immuni.2009.04.014

30. Chi, H. Regulation and function of mTOR signalling in T cell fate decisions. Nature Reviews Immunology (2012). doi:10.1038/nri3198

31. Araújo, N. C., Sampaio Gonçalves De Lucena, S. B. \& Da Silveira Rioja, S. Effect of rapamycin on spleen size in longstanding renal transplant recipients. Transplant. Proc. (2014).

1122 33. Rodrik-Outmezguine, V. S. et al. Overcoming mTOR resistance mutations with a new-generation 
mTOR inhibitor. Nature (2016). doi:10.1038/nature17963

34. Mahauad-Fernandez, W. D., Rakhra, K. \& Felsher, D. W. Generation of a tetracycline regulated mouse model of MYC-induced T-cell acute lymphoblastic leukemia. in Methods in Molecular Biology (2021). doi:10.1007/978-1-0716-1476-1_16

35. Felsher, D. W. \& Bishop, J. M. Reversible tumorigenesis by MYC in hematopoietic lineages. Mol. Cell 4, 199-207 (1999).

36. Shachaf, C. M. et al. MYC inactivation uncovers pluripotent differentiation and tumour dormancy in hepatocellular cancer. Nature (2004). doi:10.1038/nature03043

37. Casey, S. C. et al. MYC regulates the antitumor immune response through CD47 and PD-L1. Science (80-. ). (2016). doi:10.1126/science.aac9935

38. Mahauad-Fernandez, W. D., DeMali, K. A., Olivier, A. K. \& Okeoma, C. M. Bone marrow stromal antigen 2 expressed in cancer cells promotes mammary tumor growth and metastasis. Breast Cancer Res. (2014). doi:10.1186/s13058-014-0493-8

39. Li, Y., Choi, P. S., Casey, S. C., Dill, D. L. \& Felsher, D. W. MYC through miR-17-92 suppresses specific target genes to maintain survival, autonomous proliferation, and a Neoplastic state.

40. Gouw, A. M. et al. The MYC Oncogene Cooperates with Sterol-Regulated Element-Binding

41. Mahauad-Fernandez, W. D. doi:10.1016/j.cmet.2019.07.012 anoikis resistance to breast cancer cells by negating proapoptotic activities to promote tumor cell survival and growth. Cell Death Dis. (2017). doi:10.1038/cddis.2017.68

42. Fan, A. C. et al. Nanofluidic proteomic assay for serial analysis of oncoprotein activation in 1147 43. Lai, I. et al. Lipid nanoparticles that deliver IL-12 messenger RNA suppress tumorigenesis in MYC oncogene-driven hepatocellular carcinoma. J. Immunother. Cancer (2018). 
1150 44. Dhanasekaran, R. et al. MYC and twist1 cooperate to drive metastasis by eliciting crosstalk between cancer and innate immunity. Elife (2020). doi:10.7554/eLife.50731

45. Newman, A. M. et al. Robust enumeration of cell subsets from tissue expression profiles. Nat. Methods (2015). doi:10.1038/nmeth.3337

46. Newman, A. M. et al. Determining cell type abundance and expression from bulk tissues with digital cytometry. Nat. Biotechnol. (2019). doi:10.1038/s41587-019-0114-2

47. Li, T. et al. TIMER2.0 for analysis of tumor-infiltrating immune cells. Nucleic Acids Res. (2021).

48. Mortazavi, A., Williams, B. A., McCue, K., Schaeffer, L. \& Wold, B. Mapping and quantifying mammalian transcriptomes by RNA-Seq. Nat. Methods (2008). doi:10.1038/nmeth.1226

49. Zeller, K. I., Jegga, A. G., Aronow, B. J., O’Donnell, K. A. \& Dang, C. V. An integrated database targets. Genome Biol. (2003). doi:10.1186/gb-2003-4-10-r69

50. Plym Forshell, L. et al. The direct Myc target Pim3 cooperates with other Pim kinases in

51. Bueno, M. J. et al. Combinatorial effects of microRNAs to suppress the Myc oncogenic pathway. Blood (2011). doi:10.1182/blood-2010-10-315432

52. Zwolinska, A. K., Heagle Whiting, A., Beekman, C., Sedivy, J. M. \& Marine, J. C. Suppression of Myc oncogenic activity by nucleostemin haploinsufficiency. Oncogene (2012).

1173 54. Yokoi, K. et al. Survival pathway of cholangiocarcinoma via AKT/mTOR signaling to escape RAF/MEK/ERK pathway inhibition by sorafenib. Oncol. Rep. (2018). doi:10.3892/or.2017.6153

1175 55. Goltsev, Y. et al. Deep Profiling of Mouse Splenic Architecture with CODEX Multiplexed Imaging. Cell (2018). doi:10.1016/j.cell.2018.07.010 
1177 56. El-Khoueiry, A. B. et al. Nivolumab in patients with advanced hepatocellular carcinoma (CheckMate 040): an open-label, non-comparative, phase 1/2 dose escalation and expansion trial. Lancet 389, 2492-2502 (2017).

57. Sangro, B. et al. A clinical trial of CTLA-4 blockade with tremelimumab in patients with hepatocellular carcinoma and chronic hepatitis C. J. Hepatol. 59, 81-88 (2013).

58. Finn, R. S. et al. Pembrolizumab As Second-Line Therapy in Patients With Advanced Hepatocellular Carcinoma in KEYNOTE-240: A Randomized, Double-Blind, Phase III Trial. J. Clin. Oncol. 38, 193-202 (2020).

59. Ng, S. S. et al. The NK cell granule protein NKG7 regulates cytotoxic granule exocytosis and inflammation. Nat. Immunol. (2020). doi:10.1038/s41590-020-0758-6

60. Martínez-Lostao, L., Anel, A. \& Pardo, J. How Do Cytotoxic Lymphocytes Kill Cancer Cells? Clin.

61. Lemaitre, J. M., Buckle, R. S. \& Méchali, M. c-Myc in the Controlm of Cell Proliferation and

62. Pajic, A. et al. Cell cycle activation by c-myc in a Burkitt lymphoma model cell line. Int. J. Cancer (2000). doi:10.1002/1097-0215(20000915)87:6<787::AID-IJC4>3.0.CO;2-6

63. Dranoff, G. Experimental mouse tumour models: what can be learnt about human cancer

66. Rakhra, K. et al. CD4+ T Cells contribute to the remodeling of the microenvironment required for immunology? Nat. Rev. Immunol. 12, 61-66 (2011).

64. Mittal, D., Gubin, M. M., Schreiber, R. D. \& Smyth, M. J. New insights into cancer immunoediting and its three component phases--elimination, equilibrium and escape. Curr. Opin. Immunol. 27, $16-25$ (2014).

65. Schürch, C. M. et al. Coordinated Cellular Neighborhoods Orchestrate Antitumoral Immunity at the Colorectal Cancer Invasive Front. Cell (2020). doi:10.1016/j.cell.2020.07.005

1203 67. Swaminathan, S. et al. MYC functions as a switch for natural killer cell-mediated immune 
surveillance of lymphoid malignancies. Nat. Commun. 11, 2860 (2020).

1205

1206

1207

1208

1209

1210

1211

1212

1213

1214

1215

1216

1217

1218

1219

1220

1221

1222

1223

1224

1225

1226

1227

1228

1229

1230

68. Powell, J. D., Lerner, C. G. \& Schwartz, R. H. Inhibition of cell cycle progression by rapamycin induces T cell clonal anergy even in the presence of costimulation. J. Immunol. (1999).

69. Ma, L., Chen, Z., Erdjument-Bromage, H., Tempst, P. \& Pandolfi, P. P. Phosphorylation and functional inactivation of TSC2 by Erk: Implications for tuberous sclerosis and cancer pathogenesis. Cell (2005). doi:10.1016/j.cell.2005.02.031

70. Zhou, S. L. et al. Tumor-Associated Neutrophils Recruit Macrophages and T-Regulatory Cells to Promote Progression of Hepatocellular Carcinoma and Resistance to Sorafenib.

Gastroenterology 150, 1646-1658.e17 (2016).

71. Dong, N. et al. M2 macrophages mediate sorafenib resistance by secreting HGF in a feedforward manner in hepatocellular carcinoma. Br. J. Cancer 121, 22-33 (2019).

72. Chen, J. et al. Sorafenib-resistant hepatocellular carcinoma stratified by phosphorylated ERK activates PD-1 immune checkpoint. Oncotarget 7, 41274-41284 (2016).

73. Zhang, Q. B. et al. Suppression of natural killer cells by sorafenib contributes to prometastatic effects in hepatocellular carcinoma. PLoS One 8, (2013).

74. Chen, Y. et al. CXCR4 inhibition in tumor microenvironment facilitates anti-programmed death receptor-1 immunotherapy in sorafenib-treated hepatocellular carcinoma in mice. Hepatology 61, 1591-1602 (2015).

75. Zhu, H., Yang, X., Zhao, Y. \& Yi, C. Efficacy of anti-PD-1 antibody SHR-1210 as second-line treatment in hepatocellular carcinoma patient with sorafenib resistance: A case report. Medicine (Baltimore). 98, (2019).

76. Wang, Y. et al. Combinatorial immunotherapy of sorafenib and blockade of programmed deathligand 1 induces effective natural killer cell responses against hepatocellular carcinoma. Tumour Biol. 36, 1561-1566 (2015).

77. Lo, J. et al. Nuclear factor kappa B-mediated CD47 up-regulation promotes sorafenib resistance and its blockade synergizes the effect of sorafenib in hepatocellular carcinoma in mice. Hepatology 62, 534-545 (2015). 
bioRxiv preprint doi: https://doi.org/10.1101/2022.02.04.478208; this version posted February 5, 2022. The copyright holder for this preprint

(which was not certified by peer review) is the author/funder, who has granted bioRxiv a license to display the preprint in perpetuity. It is made available under aCC-BY-ND 4.0 International license.

1231 78. Casacuberta-Serra, S. \& Soucek, L. Myc and Ras, the Bonnie and Clyde of immune evasion.

1232 Transl. Cancer Res. 7, 1284-300 (2018).

1233 


\section{FIGURE 1}

a

Untreated

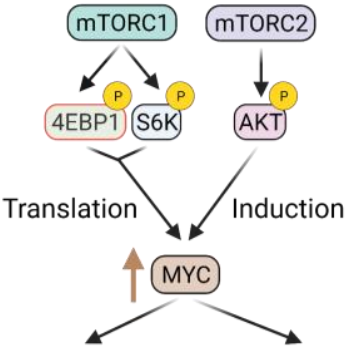

Tumor growth Immune evasion

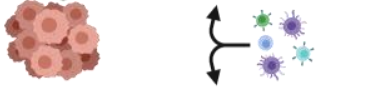

mTORC1/2 Inhibitor

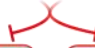

mTORC1 mTORC2

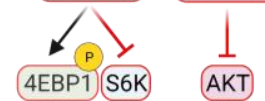

4EBP 1 S6K AKT

Translation
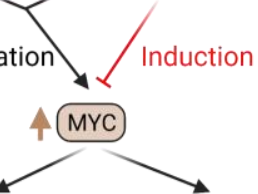

Reduced growth Immunosuppression

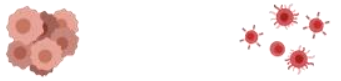

mTORC1 inhibitor
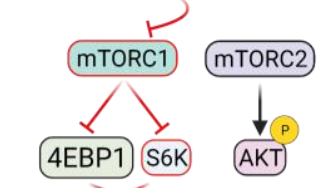

Translation
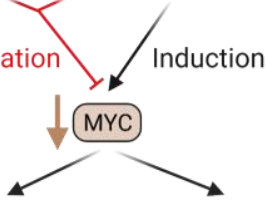

Tumor regression Immune sensitivity

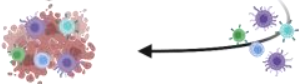

b

Selective bi-steric mTORC1 inhibitors
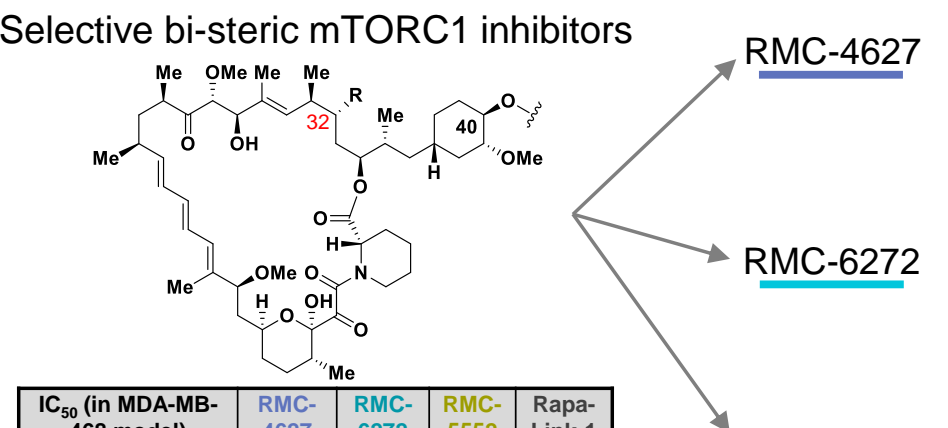

\begin{tabular}{|c|c|c|c|c|}
\hline $\begin{array}{c}\text { IC }_{50} \text { (in MDA-MB- } \\
\text { 468 model) }\end{array}$ & $\begin{array}{c}\text { RMC- } \\
4627\end{array}$ & $\begin{array}{c}\text { RMC- } \\
6272\end{array}$ & $\begin{array}{c}\text { RMC- } \\
5552\end{array}$ & $\begin{array}{c}\text { Rapa- } \\
\text { Link-1 }\end{array}$ \\
\hline $\mathrm{pS6K}(\mathrm{nM})$ & 0.25 & 0.14 & 0.14 & 0.93 \\
\hline $\mathrm{p} 4 \mathrm{EBP} 1(\mathrm{nM})$ & 1.2 & 0.44 & 0.48 & 1.73 \\
\hline $\mathrm{pAKT}(\mathrm{nM})$ & 16 & 12 & 19 & 6.32 \\
\hline $\begin{array}{c}\text { Selectivity } \\
\left.\text { (IC } \mathrm{C}_{50} \mathrm{pAKT} / \mathrm{p} 4 \mathrm{EBP} 1\right)\end{array}$ & 13 & 27 & 40 & 4 \\
\hline
\end{tabular}
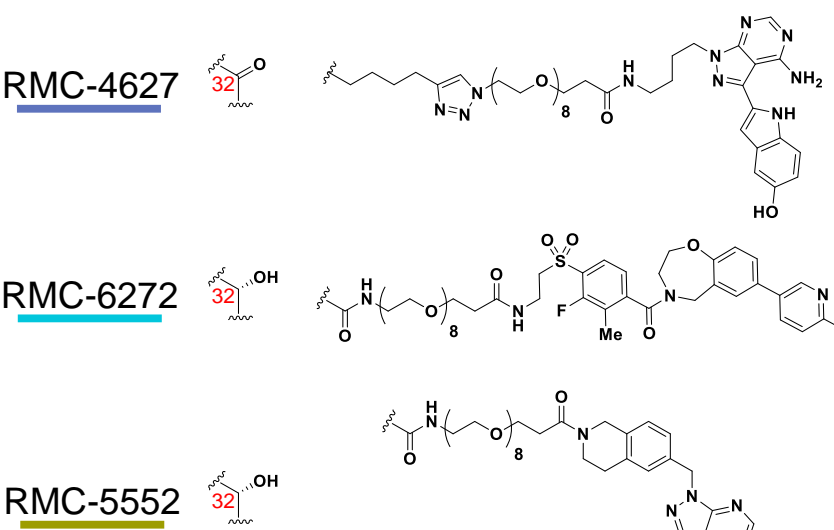

HO
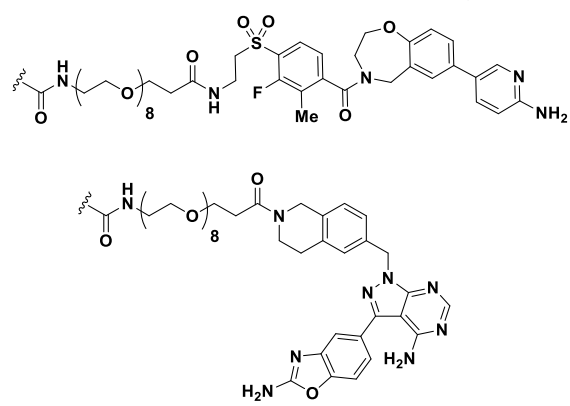

C

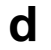

e
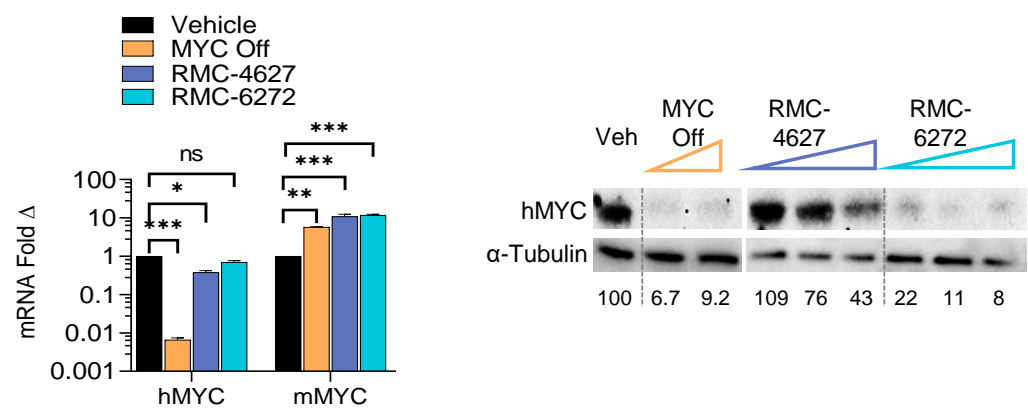

f

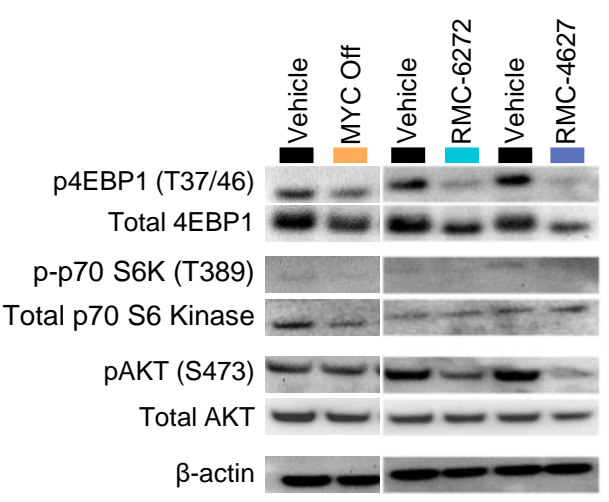

\begin{tabular}{|c|c|c|c|}
\hline \multirow{2}{*}{$\begin{array}{c}\text { Treatment } \\
\text { (in EC4 model) }\end{array}$} & \multicolumn{3}{|c|}{ Inhibition (\%) of } \\
\cline { 2 - 4 } & \multicolumn{2}{|c|}{ mTORC1 } & mTORC2 \\
\cline { 2 - 4 } p4EBP1 & pS6k & pAKT \\
\hline Doxycycline (MYC Off) & 19.83 & 7.23 & 28.82 \\
\hline RMC-4627 & 54.61 & 29.57 & 32.83 \\
\hline RMC-6272 & 45.95 & 30.20 & 12.66 \\
\hline
\end{tabular}

g

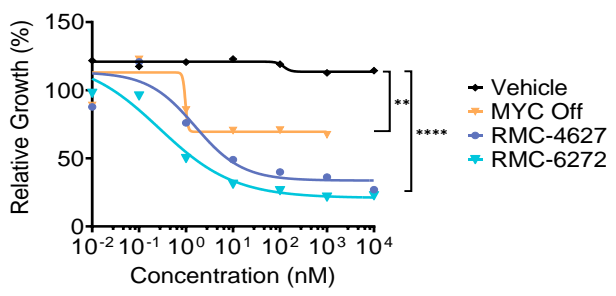

h

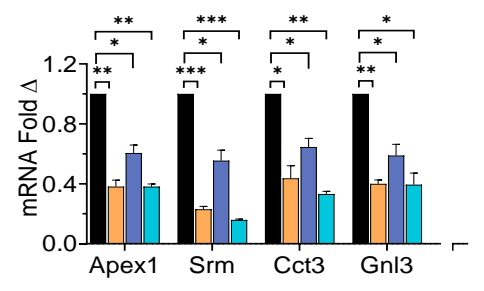


a

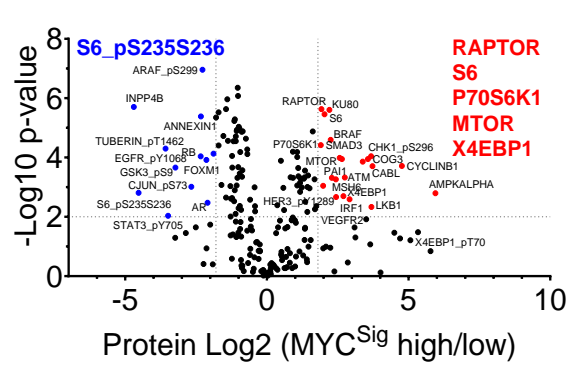

C

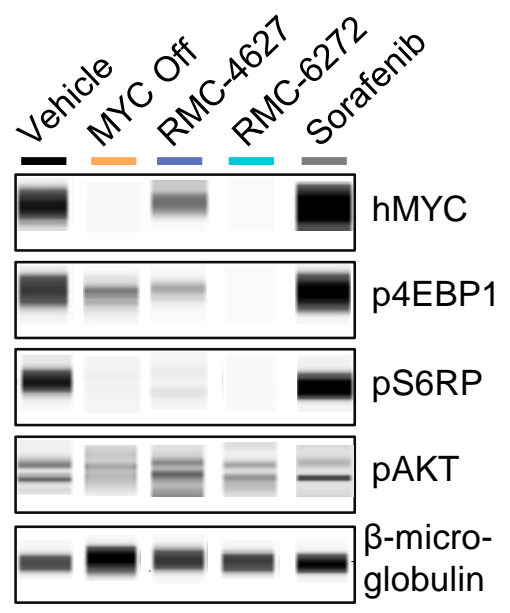

e

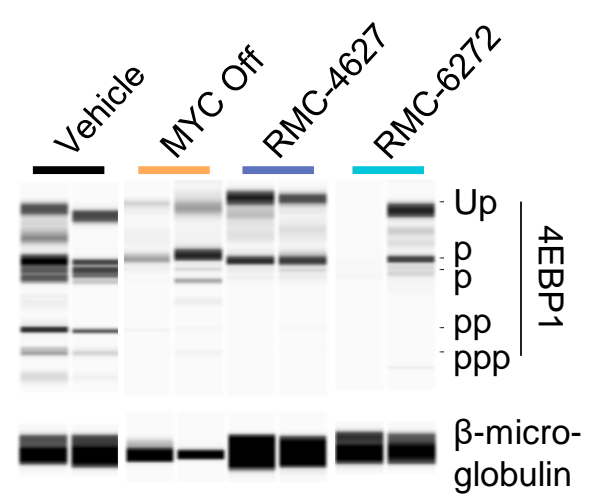

g

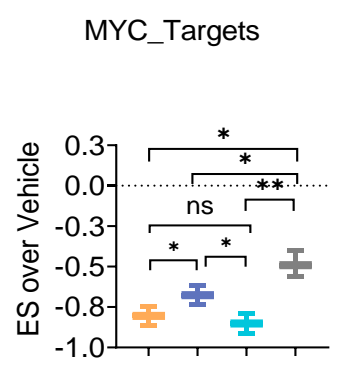

h

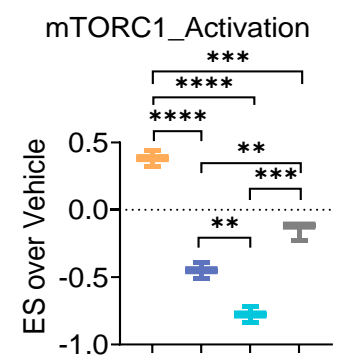

k

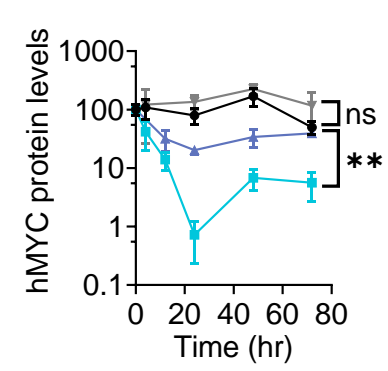

b

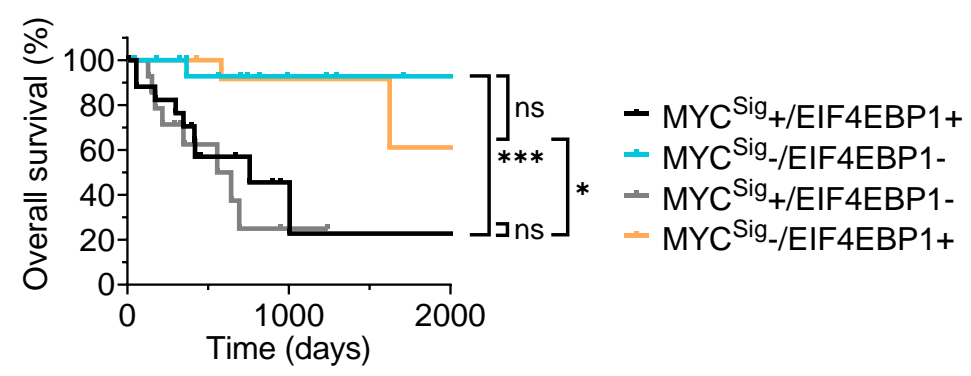

d
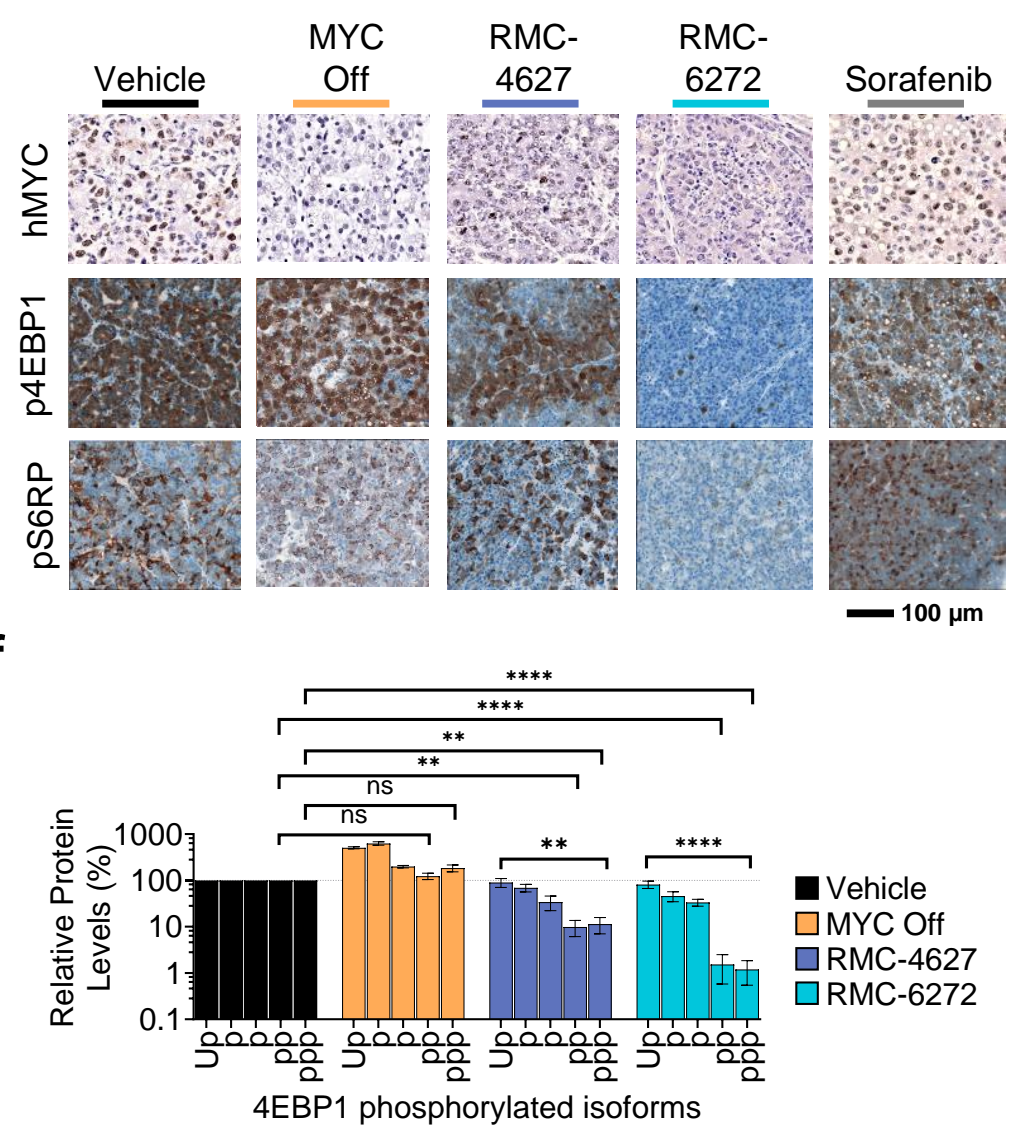

i

mTORC2_Activation

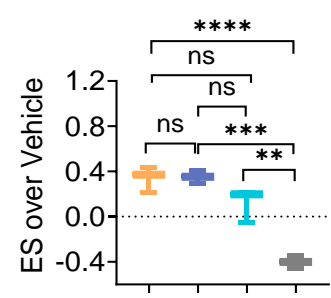

m

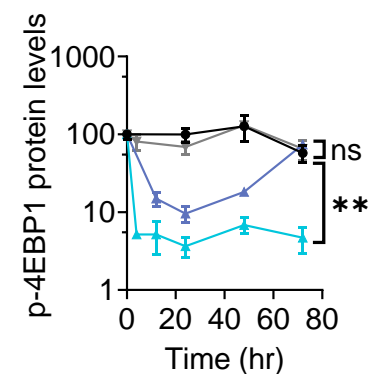

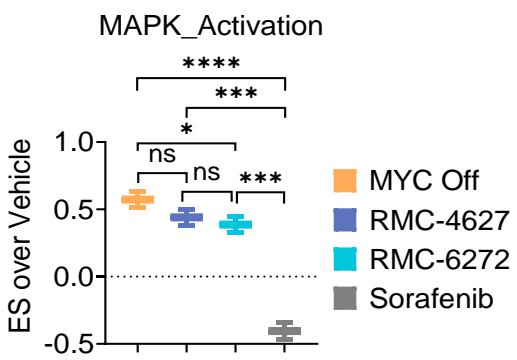

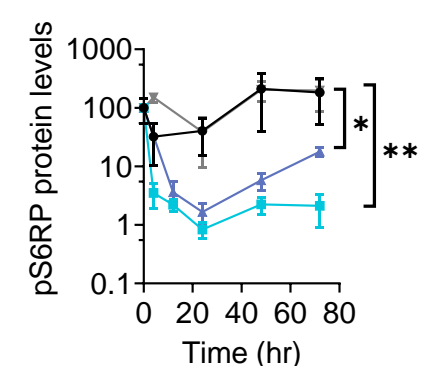

n

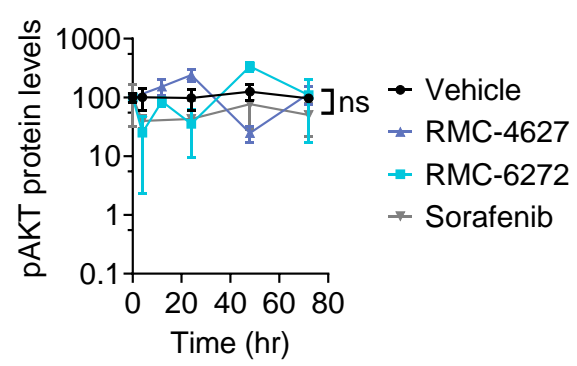


a

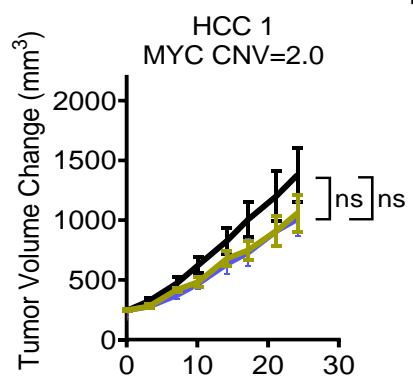

b

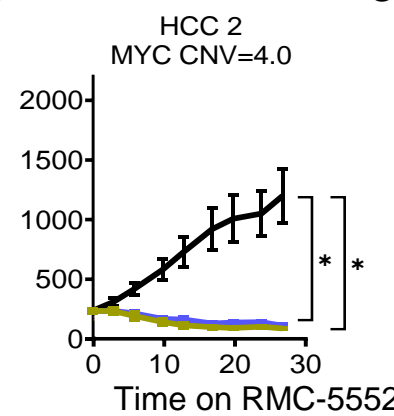

C

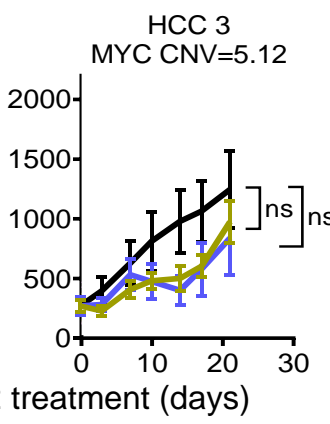

d

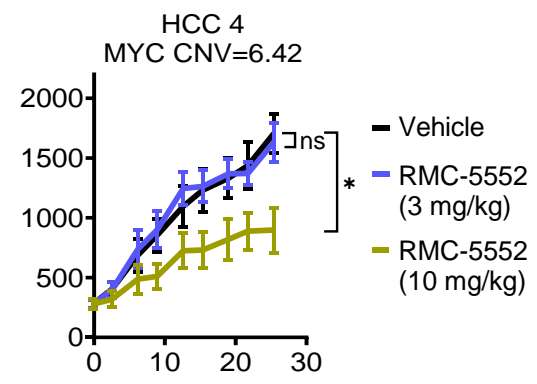

e

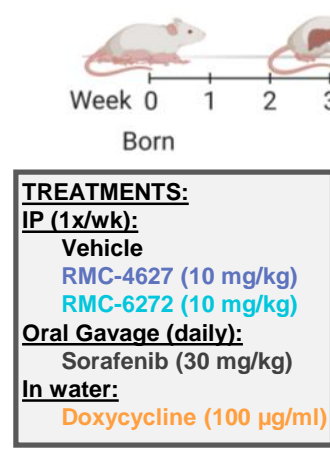

f

g

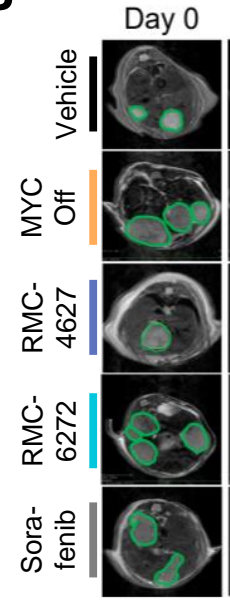

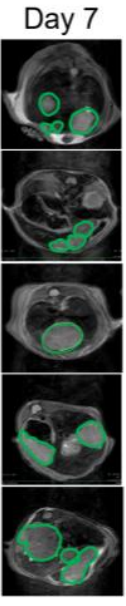

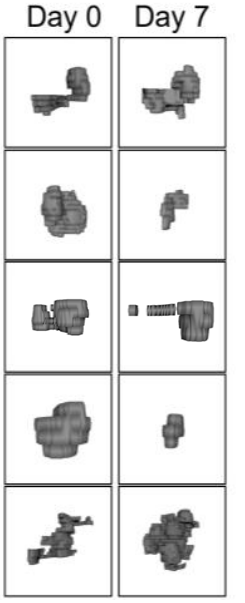

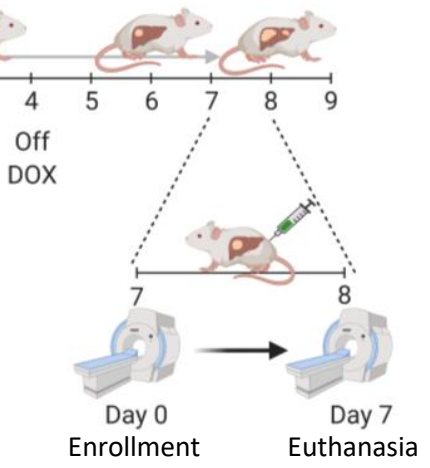

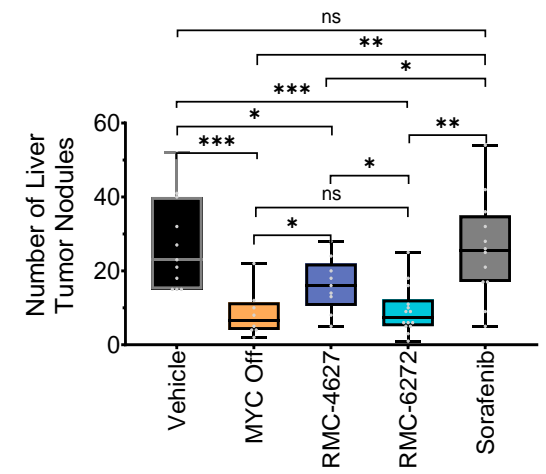

h

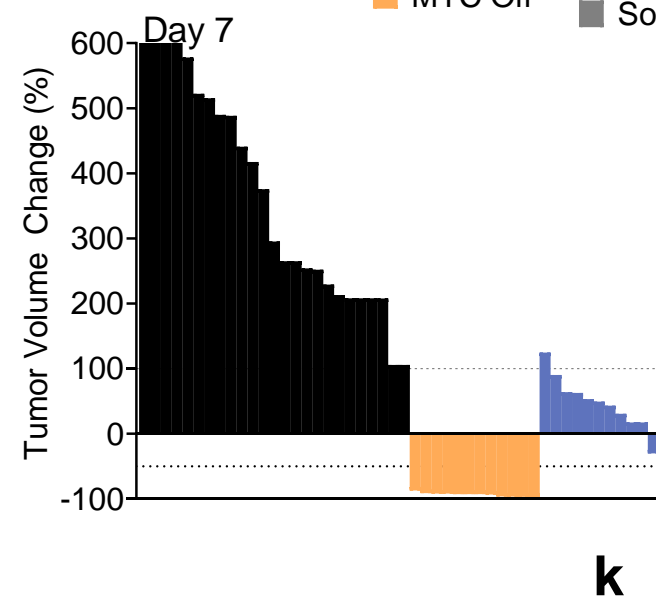

- Vehicle - RMC-4627

- MYC Off - RMC-6272

$\rightarrow$ Sorafenib

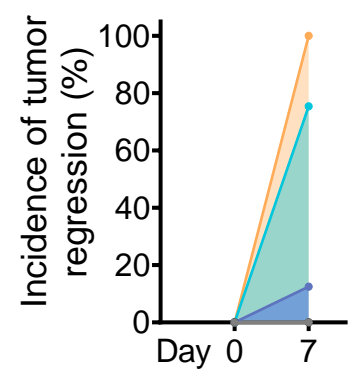

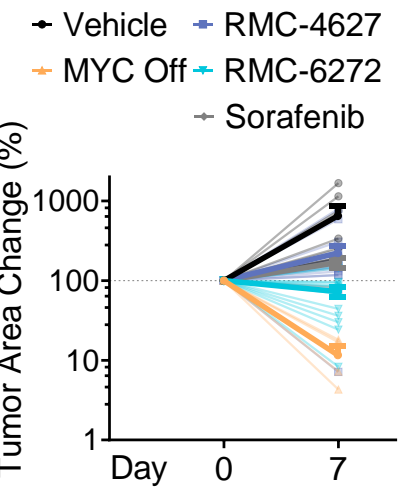

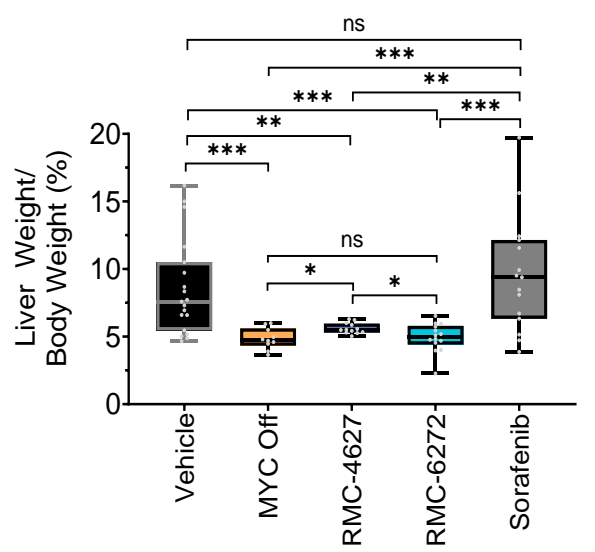




\section{FIGURE 4}

a

IMMUNE_SYSTEM_PROCESS

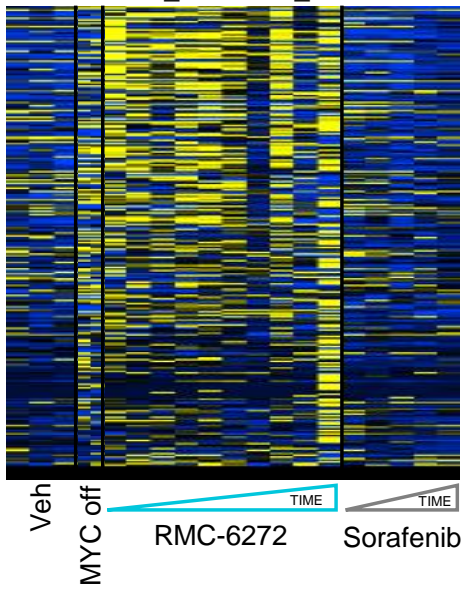

d

RMC-6272/Veh 24h

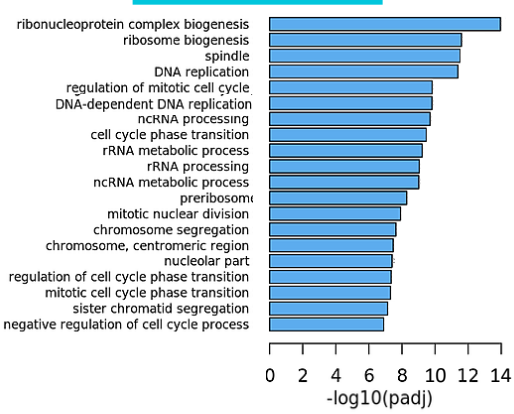

b

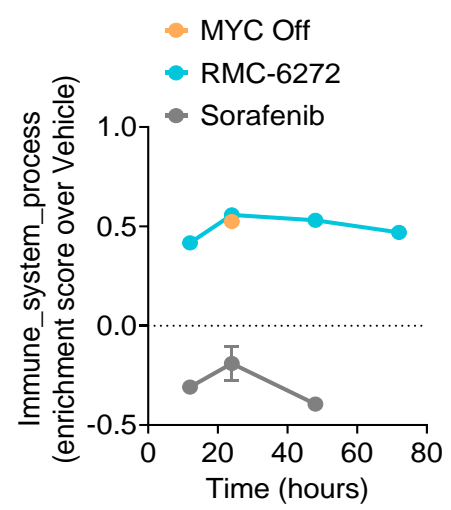

e

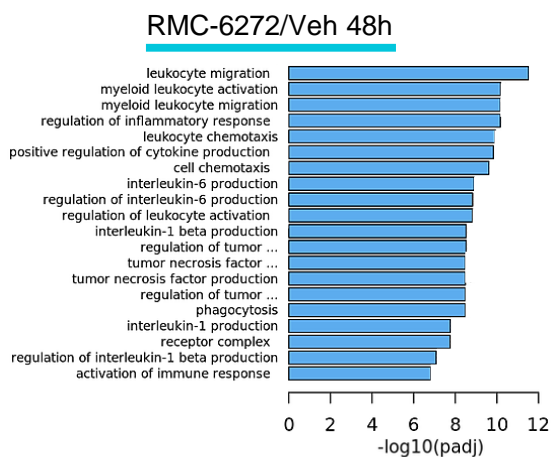

C
IMMUNE_SYSTEM_PROCESS

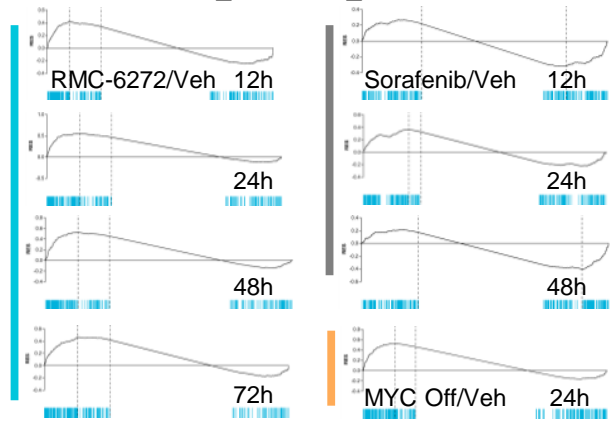

f

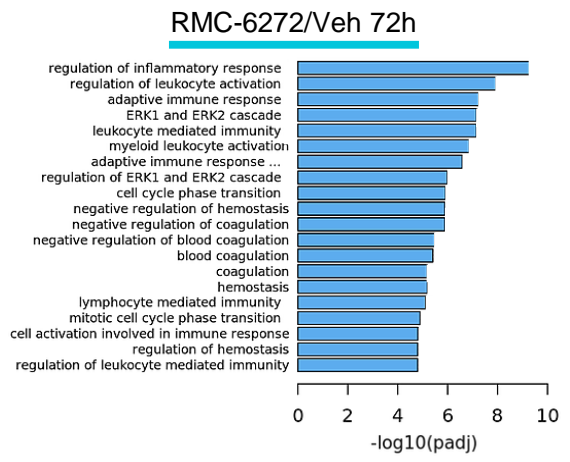

g

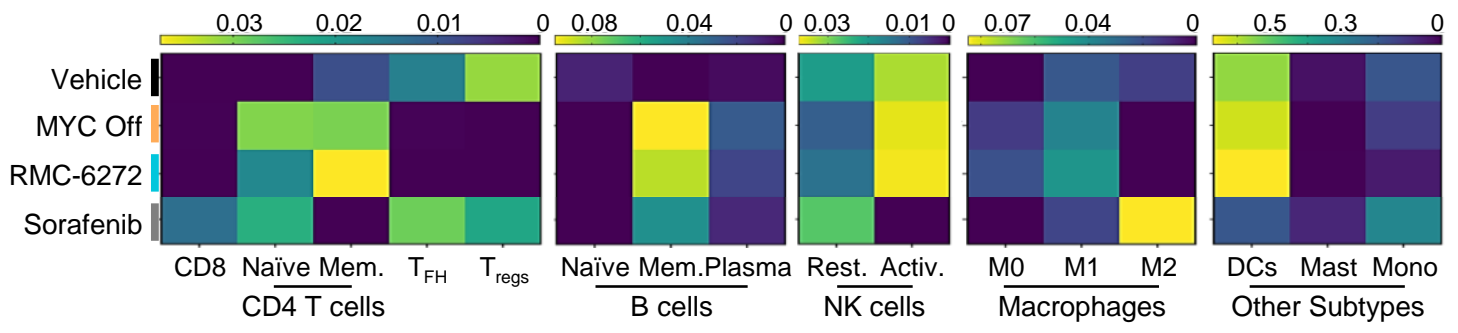

h
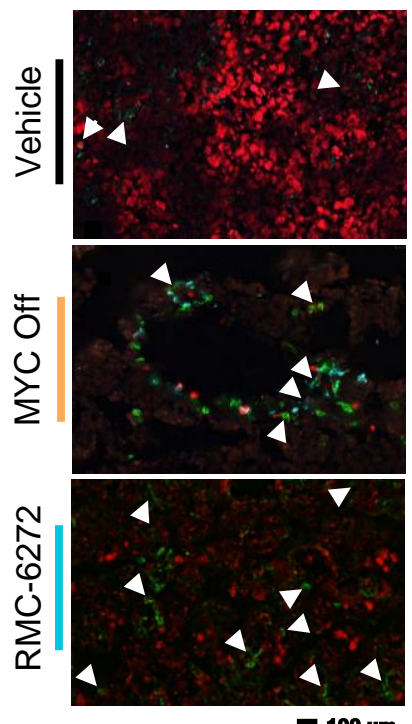

Ki67

CD4

CD8a

CD11c

CD19

i

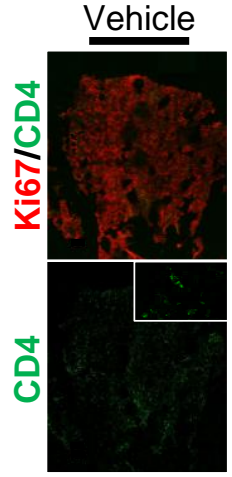

k
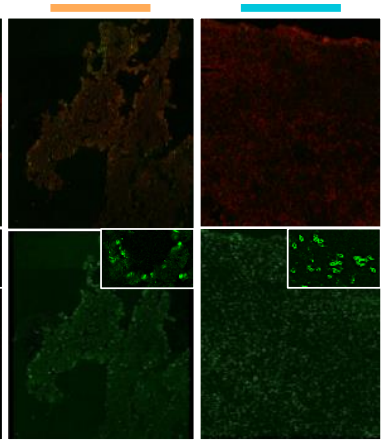

Ki67

CD8a

CD4

$\square \mathrm{CD} 11 \mathrm{C}$

$\square$ Unstained $\square$ CD19

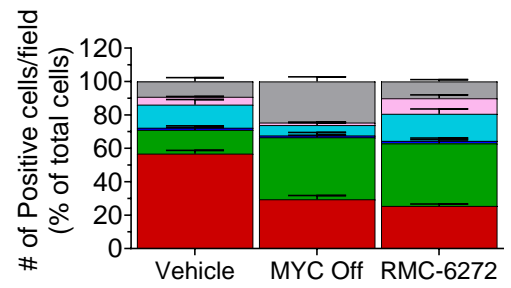

$-1 \mathrm{~mm}$

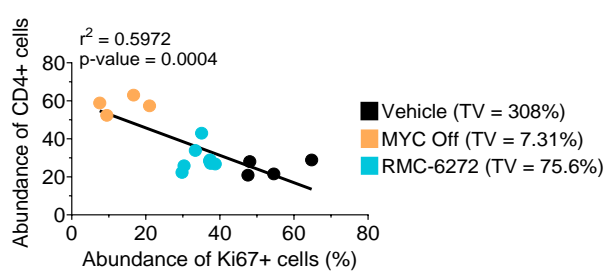


a

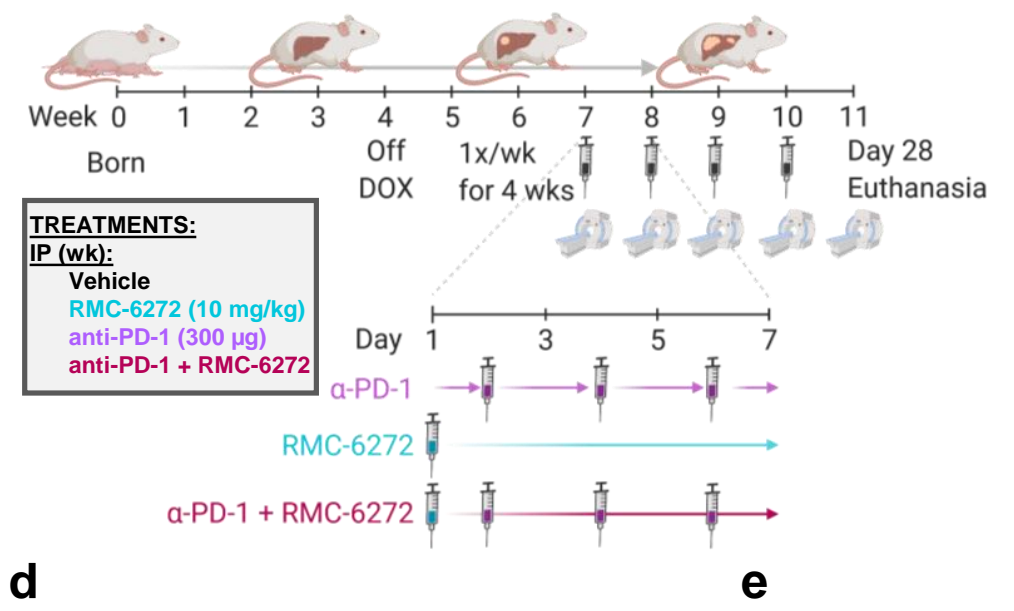

b

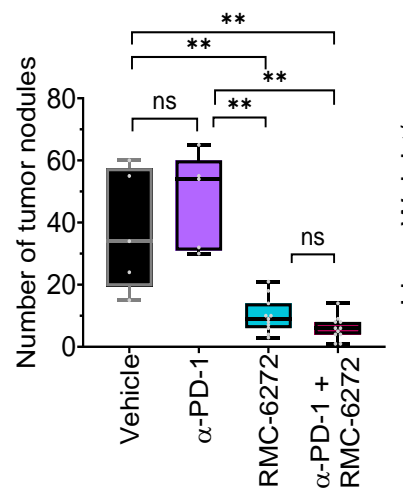

C

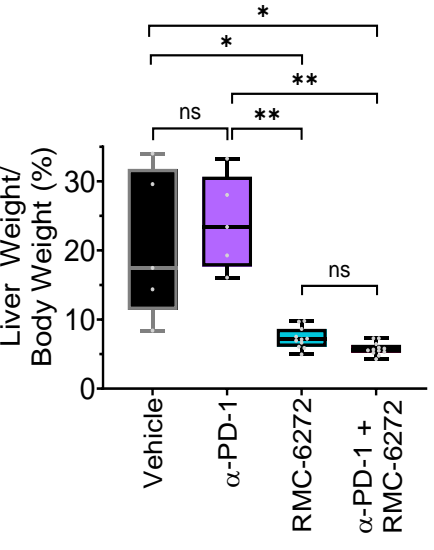

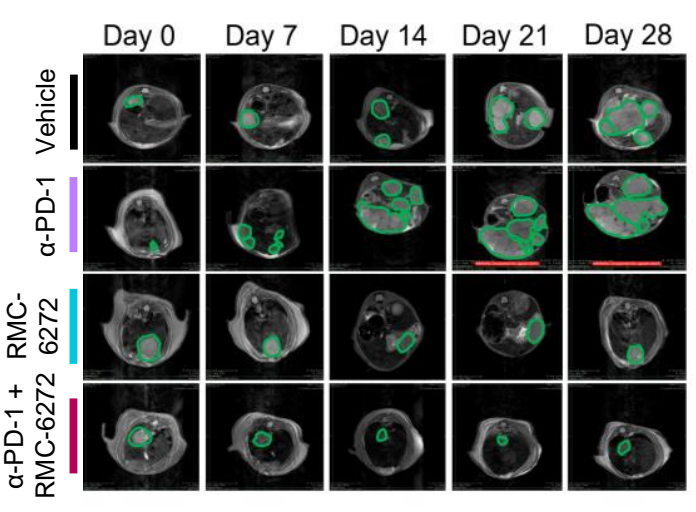

f

- Vehicle -RMC-6272

- $\alpha$-PD-1 $+\alpha$-PD-1 + RMC-6272

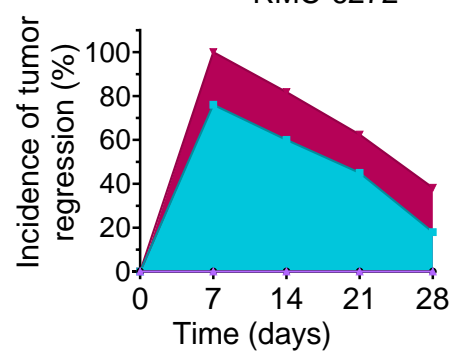

g

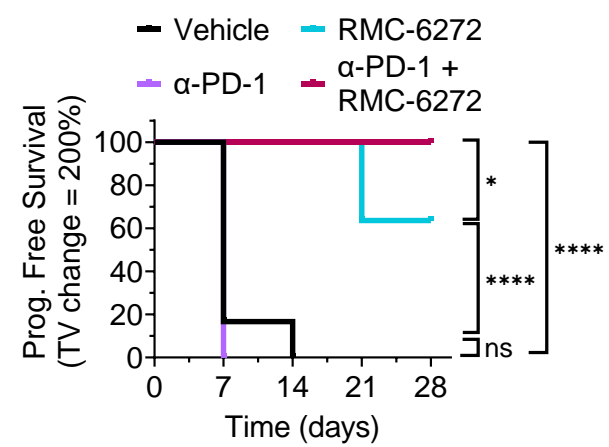

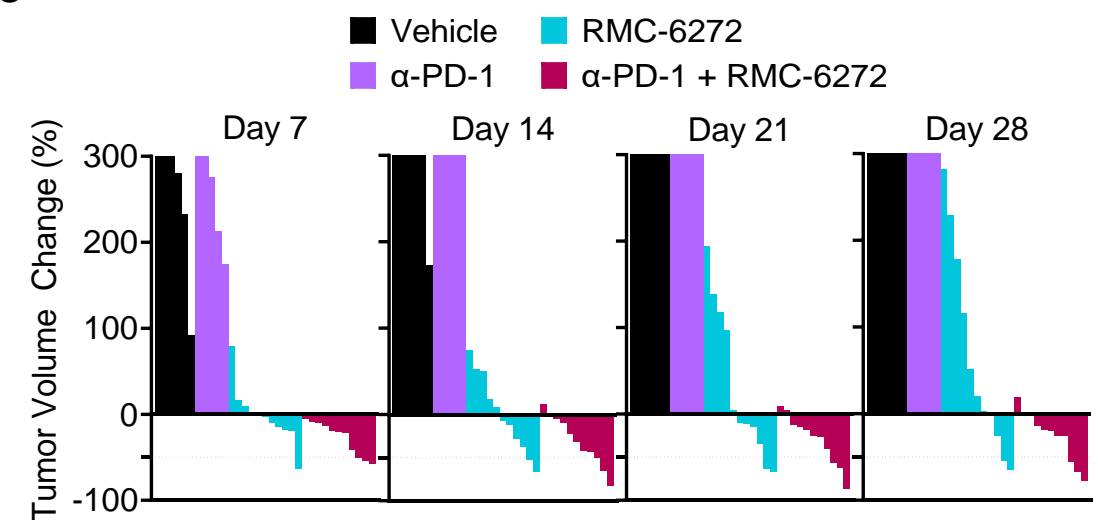

h
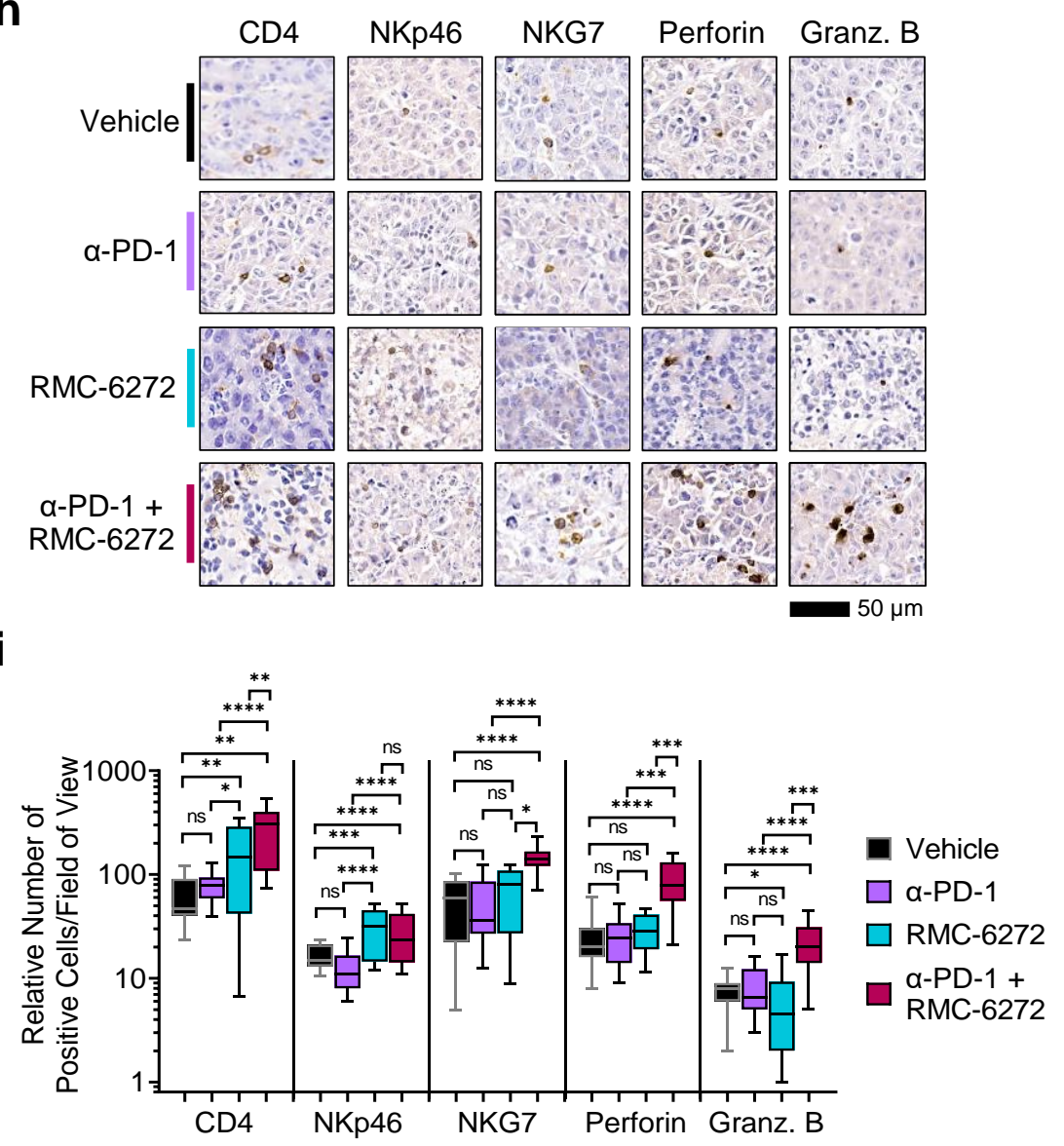

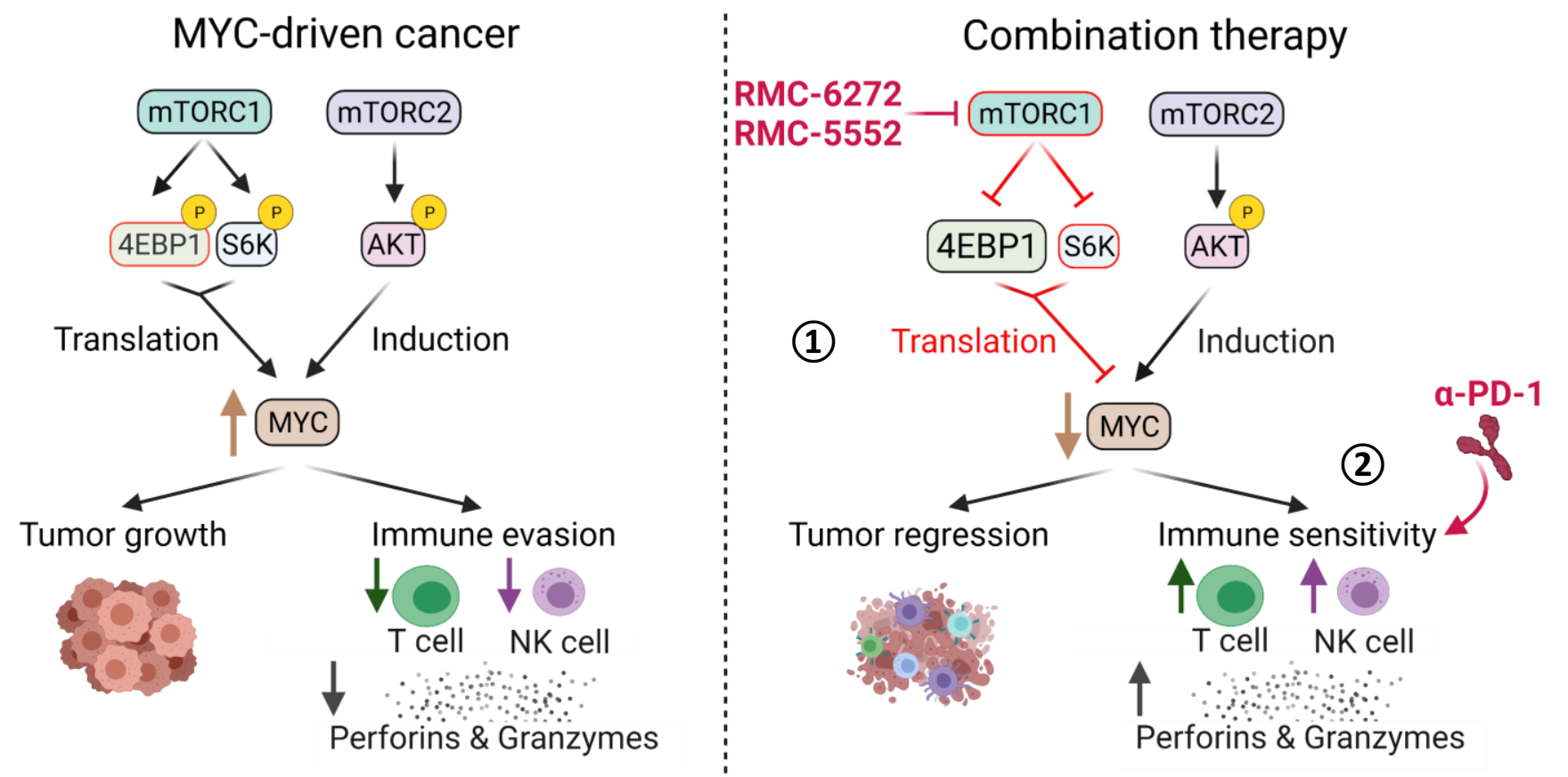